\title{
Intracellular Calcium Responses Encode Action Potential Firing in Spinal Cord Lamina I Neurons
}

\author{
Erika K. Harding, ${ }^{1,2,3}$ Bruno Boivin, ${ }^{1,4}$ and ${ }^{\circledR}$ Michael W. Salter ${ }^{1,2}$ \\ ${ }^{1}$ Program in Neurosciences \& Mental Health, The Hospital for Sick Children, Toronto, Ontario Canada M5G 0A4, ${ }^{2}$ Department of Physiology, \\ University of Toronto, Toronto, Ontario Canada M5S 1A8, ${ }^{3}$ Department of Comparative Biology and Experimental Medicine, University of Calgary, \\ Calgary, Alberta Canada T2N 4N1, and ${ }^{4}$ F.M. Kirby Neurobiology Center, Boston Children's Hospital, Harvard Medical School, Boston, \\ Massachusetts 02115
}

Maladaptive plasticity of neurons in lamina I of the spinal cord is a lynchpin for the development of chronic pain, and is critically dependent on intracellular calcium signaling. However, the relationship between neuronal activity and intracellular calcium in these neurons is unknown. Here we combined two-photon calcium imaging with whole-cell electrophysiology to determine how action potential firing drives calcium responses within subcellular compartments of male rat spinal cord lamina I neurons. We found that single action potentials generated at the soma increase calcium concentration in the somatic cytosol and nucleus, and these calcium responses invade dendrites and dendritic spines by active backpropagation. Calcium responses in each compartment were dependent on voltage-gated calcium channels, and somatic and nuclear calcium responses were amplified by release of calcium from ryanodine-sensitive intracellular stores. Grouping single action potentialevoked calcium responses by neuron type demonstrated their presence in all defined types, as well as a high degree of similarity in calcium responses between neuron types. With bursts of action potentials, we found that calcium responses have the capacity to encode action potential frequency and number in all compartments, with action potential number being preferentially encoded. Together, these findings indicate that intracellular calcium serves as a readout of neuronal activity within lamina I neurons, providing a unifying mechanism through which activity may regulate plasticity, including that seen in chronic pain.

Key words: calcium imaging; dendrites; dendritic spines; lamina I; nucleus; spinal cord

Significance Statement

Despite their critical role in both acute pain sensation and chronic pain, little is known of the fundamental physiology of spinal cord lamina I neurons. This is especially the case with respect to calcium dynamics within these neurons, which could regulate maladaptive plasticity observed in chronic pain. By combining two-photon calcium imaging and patch-clamp electrophysiological recordings from lamina I neurons, we found that action potential firing induces calcium responses within the somatic cytosol, nucleus, dendrites, and dendritic spines of lamina I neurons. Our findings demonstrate the presence of actively backpropagating action potentials, shifting our understanding of how these neurons process information, such that calcium provides a mechanism for lamina I neurons to track their own activity.

\section{Introduction}

Lamina I neurons of the spinal cord form an integrative hub in the path of nociceptive information from the periphery to the brain. Neurons in this outermost spinal cord layer receive direct

Received Jan. 22, 2020; revised Apr. 5, 2020; accepted Apr. 19, 2020.

Author contributions: E.K.H. and M.W.S. designed research; E.K.H. performed research; E.K.H. analyzed data; E.K.H. and M.W.S. wrote the first draft of the paper; E.K.H. and M.W.S. edited the paper; E.K.H., B.B., and M.W.S. wrote the paper; B.B. contributed unpublished reagents/analytic tools.

This work was supported by Canadian Institutes of Health Research Grant FDN-154336, and a Canadian Institutes of Health Research Frederick Banting and Charles Best Canada Graduate Scholarship Doctoral Award. The authors declare no competing financial interests.

Correspondence should be addressed to Michael W. Salter at michael.salter@sickkids.ca or Erika K. Harding at erika.harding@ucalgary.ca.

https://doi.org/10.1523/JNEUROSCI.0206-20.2020

Copyright $\odot 2020$ the authors nociceptive primary afferent inputs, which are processed by a network of excitatory and inhibitory interneurons, as well as indirect mechanosensory information via input from neurons in other layers of the spinal cord (Basbaum et al., 2009). In addition to interneurons, lamina I contains a set of projection neurons with axons that ascend to regions in the brain where nociceptive information becomes integrated into our pain experience (Gauriau and Bernard, 2002). Dysfunctional alterations in lamina I neurons have been implicated in the development of chronic pain (Mantyh et al., 1997; Coull et al., 2003; Ikeda et al., 2003; Keller et al., 2007), such that lamina I neurons in animal models of chronic pain exhibit a form of maladaptive synaptic plasticity (Ikeda et al., 2003; Ji et al., 2003; Costigan et al., 2009).

One of the major regulators of synaptic plasticity throughout the CNS is intracellular calcium, which acts as a critical second 
messenger linking excitatory synaptic transmission and neuronal firing to various downstream processes, including regulating gene transcription, receptor trafficking, and receptor and protein phosphorylation (Berridge et al., 2000; Bengtson and Bading, 2012; Bourinet et al., 2014). A major source of activity-dependent intracellular calcium in neurons are voltage-gated calcium channels (VGCCs). VGCCs are present in the axons and somata of neurons but may also be present in the dendritic arbor and dendritic spines, at the site of synapses (Sabatini et al., 2002; Higley and Sabatini, 2012; Cichon and Gan, 2015). VGCC-mediated calcium entry into dendrites and dendritic spines can affect short-term plasticity through facilitating temporal and spatial summation, and long-lasting plasticity through altering gene transcription. Critical for these changes is the firing of action potentials (APs) in the neuronal soma and the propagation of these APs into dendrites and spines, as has been well described in brain regions such as the hippocampus (Spruston et al., 1995; Rozsa et al., 2004; Kuczewski et al., 2008; Evstratova et al., 2011).

For neurons in lamina I of the spinal cord, our understanding of the relationship between AP firing and intracellular calcium is rudimentary. Depolarizing lamina I neurons with stimuli, such as bath application of glutamate or bursts of APs, are known to raise calcium concentration in the soma (Ikeda et al., 2003; Luo et al., 2008; Doolen et al., 2012). However, there is no information on how frequency or pattern of APs, which are key parameters for synaptic plasticity in the CNS, affects intracellular calcium. Moreover, studies on intracellular calcium in dendrites and dendritic spines of lamina I neurons are nonexistent. Therefore, there is a major gap in our understanding of the fundamental biology of this crucial neuronal cell type. Here, we closed this gap by combining patch-clamp electrophysiology with two-photon calcium imaging to define the dynamics of APevoked calcium responses in lamina I neurons, to characterize the responses in somata, dendrites, and spines of these neurons, and to determine the mechanisms through which these calcium responses occur.

\section{Materials and Methods \\ Animals}

Animal experiments were approved by the Hospital for Sick Children Animal Care Committee and performed in accordance with animal care regulation and policies of the Canadian Council on Animal Care. All spinal cord experiments were completed using male adult (P60-P90) Sprague Dawley rats (325-400 g). All hippocampal experiments were completed using both male and female adult C57Bl/6 mice (P25-P30).

\section{Spinal cord isolation}

Male Sprague Dawley rats were anesthetized with 20\% (w/v) urethane, administered via intraperitoneal injection, and killed by severing of the cervical spinal cord and vertebrae. The lumbar region of the spinal cord was dissected from the rat, placed in a protective sucrose dissection solution containing $1 \mathrm{~mm}$ kynurenic acid, and bubbled with $5 \% \mathrm{CO}_{2} / 95 \%$ $\mathrm{O}_{2}$, pH 7.3 (310 mOsm). Dorsal roots and dura were removed from the lumbar cord, and L4-L6 were isolated from the rest of the cord. The L4L6 cord was then glued against an agar block and placed in a VT 1000s vibratome (Leica Microsystems) containing ice-cold sucrose dissection solution. Dissection solution contained the following (in $\mathrm{mM}$ ): 50 sucrose, $92 \mathrm{NaCl}, 15 \mathrm{D}$-glucose, $26 \mathrm{NaHCO}_{3}, 2.5 \mathrm{KCl}, 1.25 \mathrm{NaH}_{2} \mathrm{PO}_{4}, 0.5$ $\mathrm{CaCl}_{2}, 7 \mathrm{MgSO}_{4}, 1$ kynurenic acid, bubbled with $5 \% \mathrm{CO}_{2} / 95 \% \mathrm{O}_{2}, \mathrm{pH}$ 7.3 (310 mOsm) (Hildebrand et al., 2011, 2014); 300- $\mu \mathrm{m}$-thick parasagittal slices were obtained from the vibratome. Slices were then incubated in dissection solution at $34^{\circ} \mathrm{C}$ for $40 \mathrm{~min}$ and then cooled passively to room temperature $\left(21^{\circ} \mathrm{C}-22^{\circ} \mathrm{C}\right.$ for $\left.\geq 30 \mathrm{~min}\right)$ before electrophysiological recording and imaging.

\section{Hippocampus isolation}

Adult mice (C57Bl/6) were anesthetized with $20 \%$ (w/v) urethane (i.p. injection) and killed via decapitation. The brain was removed from the skull, and the brain was mounted on a vibratome disk and placed in the vibratome chamber with dissection solution as follows (in $\mathrm{mM}$ ): 124 $\mathrm{NaCl}, 11 \mathrm{D}$-glucose, $26 \mathrm{NaHCO}_{3}, 2.5 \mathrm{KCl}, 1.25 \mathrm{NaH}_{2} \mathrm{PO}_{4}, 2 \mathrm{CaCl}_{2}, 2$ $\mathrm{MgCl}_{2}$, bubbled with $5 \% \mathrm{CO}_{2} / 95 \% \mathrm{O}_{2}$, pH 7.3 (310 mOsm); 300- $\mu$ mthick slices were obtained from the vibratome and placed in a holding chamber at $30^{\circ} \mathrm{C}$ for $40 \mathrm{~min}$ and then cooled passively to room temperature $\left(21^{\circ} \mathrm{C}-22^{\circ} \mathrm{C}\right.$ for $\left.\geq 30 \mathrm{~min}\right)$ before placement under the microscope for electrophysiology and imaging.

\section{Electrophysiology of lamina I spinal cord and hippocampal CA1 pyramidal neurons}

Slices were placed under an AxioExaminer Z1 microscope (Carl Zeiss Microscopy), and neurons were visualized under IR-DIC optics. Lamina I neurons were delineated as those neurons present in the darker, striate region directly above the substantia gelatinosa that defines lamina II and directly below the outer white matter tracts (Chery et al., 2000; Hildebrand et al., 2014). Hippocampal CA1 pyramidal neurons were delineated by their pyramidal somatic shape and location in the cornu ammonis. Patch-clamp recordings were made with recording pipettes of 7-12 M $\Omega$, pulled by a Sutter P97 puller (Sutter Instruments). The external recording solution (ACSF) consisted of the following (mM): 125 $\mathrm{NaCl}, 20 \mathrm{D}$-glucose, $26 \mathrm{NaHCO}_{3}, 3 \mathrm{KCl}, 1.25 \mathrm{NaH}_{2} \mathrm{PO}_{4}, 2 \mathrm{CaCl}_{2}$, and 1 $\mathrm{MgCl}_{2}$, pH 7.3 (310 mOsm). Perfusion speed was $0.5 \mathrm{ml} / \mathrm{min}$ for spinal cord and $2 \mathrm{ml} / \mathrm{min}$ for hippocampus. The internal current-clamp patch pipette solution consisted of the following $(\mathrm{mM})$ : $112 \mathrm{~K}$-gluconate, 8 $\mathrm{KCl}, 10 \mathrm{HEPES}, 4 \mathrm{Mg}$-ATP, $0.3 \mathrm{Na}_{2}$-ATP, 10 phosphocreatine, 0.3 EGTA, 0.04 Alexa Fluor-594 (AF-594), 0.11 Oregon Green Bapta-1 (OGB-1), pH 7.3 (300 mOsm). A calcium calibration curve was performed, and somatic cytosolic calcium concentration in lamina I neurons was estimated to be $40-50 \mathrm{~nm}$ at resting membrane potential based on the calcium calibration curve (Tsien and Pozzan, 1989). Internal and external solutions were the same for both types of neurons. Neurons were allowed to dialyze for 20-30 min before imaging to allow the fluorescent dye concentration to equilibrate. Baseline calcium responses typically required $20 \mathrm{~min}$ to collect, and drug wash-ins were $20 \mathrm{~min}$ as well. Postdrug wash-in responses also typically took $20 \mathrm{~min}$ to collect, for a total typical recording time of $80-90 \mathrm{~min}$. All recordings were performed between $24^{\circ} \mathrm{C}$ and $28^{\circ} \mathrm{C}$.

Patch-clamp recordings were made using a Multiclamp 700B amplifier (Molecular Devices) and a Digidata 1440A Digitizer (Molecular Devices) connected to a desktop computer with pClamp 10.4 software. Current-clamp recordings were digitized at $10 \mathrm{kHz}$ and low-pass filtered at $2 \mathrm{kHz}$. The junction potential for current clamp was calculated as $14.4 \mathrm{mV}$ (i.e., $-50 \mathrm{mV}=-64.4 \mathrm{mV}$ ) (Junction potential calculator, Clampex) and has been accounted for in all experimental data. Recordings were included for analysis only if they maintained an access resistance $<45 \mathrm{M} \Omega$ and if they maintained a resting membrane potential $<-50 \mathrm{mV}$ without current injection. Bridge balance and pipette capacitance compensation were performed in all neurons. In all experiments, neurons were held between -70 and $-80 \mathrm{mV}$ (typically 0 to $-20 \mathrm{pA}$ holding current injection). Single APs were evoked with a $5 \mathrm{~ms}$ current injection at $100 \%$ success threshold. Current injection threshold was determined for each recorded neuron by test current injections in steps increasing by $20 \mathrm{pA}$, and was typically between 150 and $400 \mathrm{pA}$ for a $5 \mathrm{~ms}$ current injection. Each recording was $15 \mathrm{~s}$ in duration, with the experimental current injection at $2 \mathrm{~s}$ into recording. A minimum of $15 \mathrm{~s}$ was given between the end of one recording and the beginning of the next to allow the neuron to return to baseline. Thus, the average stimulus rate was $0.033 \mathrm{~Hz}$. Some experiments also required bursts of 2-4 APs induced at variable frequencies. This was elicited through $5 \mathrm{~ms}$ current injections at different frequencies $(0.5,10,50 \mathrm{~Hz})$. Some experiments involved long depolarizations of $500-1000 \mathrm{~ms}$. For these experiments, neurons were held at $-70 \mathrm{mV}$; then step injections of current were elicited every $10 \mathrm{~s}$. Current injection steps started at $100 \mathrm{pA}$ below baseline and ramped up by $50 \mathrm{pA}$ to a maximum step of $100-150 \mathrm{pA}$ above baseline. These experiments were used to determine lamina I neuron firing 
type. Recordings between hippocampal and spinal cord slices were performed side by side, with alternating preparations on alternating days. All internal and external recording solutions were the same for both preparations.

\section{Two-photon calcium imaging of lamina I spinal cord and hippocampal CA1 pyramidal neurons}

Two-photon calcium imaging was performed on a 710 NLO system equipped with an AxioExaminar Z1 (Carl Zeiss Microscopy). Two-photon dual excitation of OGB-1 (110 $\mu \mathrm{M}$ in recording pipette) and AF-594 (35 $\mu \mathrm{M}$ in recording pipette) was achieved using a Coherent Chameleon Ultra Ti:Sapphire laser tuned to $800 \mathrm{~nm}$ (Coherent). We chose these concentrations based on concentrations used in previous studies (Denk et al., 1996; Maravall et al., 2000; Jia et al., 2011). OGB-1 and AF-594 fluorescence were split based on emission spectra using NDD filter cubes (500-550 nm, 565-610 nm; Carl Zeiss Microscopy), and sent into NDD detectors (Carl Zeiss Microscopy). Two-photon images were obtained using a $20 \times$ waterimmersion objective lens (Carl Zeiss Microscopy) and the Zen 2009 acquisition program (Carl Zeiss Microscopy). Laser power was kept between $0.3 \%$ and $0.7 \%$, and gain was restrained to $650-850$ for all calcium imaging experiments. Fluorescence data were acquired using line scan acquisition $(1024 \times 1,2 \times$ averaging $)$ at a rate of $133 \mathrm{~Hz}$. Somatic line selections were selected to go directly through the middle of the soma, capturing cytosolic and nuclear compartments. Cytosolic and nuclear components of line scans were differentiated based on increased intensity of the red structural dye (AF-594) in the nucleus. We confirmed this observation with DAPI staining of the nucleus after recording, with the area of increased AF-594 intensity overlapping with that of the DAPI stain (Hardingham et al., 1997; Bengtson and Bading, 2012; Doerner et al., 2012). Based on this observation, line scans were subsequently separated into ROIs aligning to the nuclear and cytosolic compartments. Dendritic line selections were typically chosen from a random primary dendrite, $20-40 \mu \mathrm{m}$ from the soma, with a selection of experiments performed at varying distances from the soma. At the end of each recording, a $z$ stack was taken to note morphology of each neuron. These were performed at $0.8 \%-0.9 \%$ laser power with a gain of 800-950, restricted to the red channel. Fluorescence data were collected and saved as Laser Scanning Microscopy files from Zen 2009 (Carl Zeiss Microscopy).

\section{Pharmacology}

Oregon Green Bapta-1 hexapotassium salt (OGB-1) and AF-594 were obtained from Invitrogen. Ryanodine was obtained from Abcam. TTX was obtained from Alomone Labs. All other compounds were obtained from Sigma Millipore. Ryanodine was dissolved in 100\% DMSO as per instructions, for a final concentration of $>0.1 \%$ DMSO in recording ACSF; all other compounds were dissolved in distilled water and added to the recording ACSF during experiments. Pharmacological agents were added to the recording ACSF after baseline measurements and allowed to incubate the slice for 15-20 min to allow for full pharmacological effect.

\section{Raw fluorescence data analysis}

$z$ stacks were deconvolved and analyzed in Volocity (PerkinElmer). Fluorescence data were exported from Zen as Laser Scanning Microscopy files and imported into MATLAB (MathWorks) for analysis. Analysis was performed with a custom-made toolbox (Calcium Imaging Analysis Toolbox [CIAT]). Fluorescence data for each line scan recording consisted of red and green intensity values for each pixel at every time point, creating a pixel $\times$ time $\times$ color matrix. Using CIAT, the pixel $\times$ time matrices for each color were averaged separately over several trials. This produced an average pixel intensity $\times$ time matrix for each color. Then, ROIs aligning to the desired compartment were delineated using a marquee function, and the pixel dimension was collapsed by averaging all pixels at each given time point together within the ROI. $\Delta \mathrm{G} / \mathrm{R}$ was then calculated from average pixel intensity, with the first $2 \mathrm{~s}$ before the AP acting as the baseline fluorescence, and dividing by the red non-calcium-sensitive control channel, creating an instantaneous calcium response curve.
This process was performed for every compartment represented in the matrix (e.g., cytosol and nucleus are both contained within a single somatic matrix). We noted a subset of neurons with photobleaching in the red, but not green, channel, which resulted in apparent run-up of the $\Delta \mathrm{G} / \mathrm{R}$ signal. We developed a subroutine within CIAT to delineate and subtract photobleaching from the data, by fitting the red channel to an exponential decay function, and removing this decay if present. Specifically, we fitted the data to a two-term exponential function of the form $f(x)=a \times \exp (b \times x)+c \times \exp (d \times x)$. Because this equation has 2 terms, we were able to separate the constant part from the exponential decay, the latter which we removed to correct for photobleaching. We also developed a subroutine to automatically remove traces with spontaneous calcium responses above a user-determined threshold (likely due to synaptic calcium responses).

CIAT was programmed to calculate the time to peak (measured as the time from AP initiation to the peak, determined from 10-point smoothed data to reduce noise), and peak response (measured as the $50 \mathrm{~ms}$ average around the maximum value of the curve, constrained to between 0 and $3 \mathrm{~s}$ after AP initiation). All data from CIAT were saved into a queryable database, as delineated by user input into the CIAT graphical user interface. The database was formatted to export data from each experiment in CIAT into 6 separate tables as follows: raw green intensity values, raw red intensity values, $\Delta \mathrm{G} / \mathrm{R}$ values, the files included for each analysis, the equation of photobleaching correction, and calculated statistics of the calcium response.

\section{Code accessibility}

Custom MATLAB code for CIAT as described above is available on Github at https://github.com/brunoboivin/ciat.

\section{Experimental design and statistical analysis}

Unless otherwise indicated, all instantaneous calcium response curves are an average of 2-8 sequential trials, as described above. Each $N$ indicates one neuron from a separate animal, unless otherwise described within the figure legends. Four main measures were typically compared for each instantaneous calcium response curve as follows: time to peak, peak response amplitude, AUC, and the decay time constant $(\tau)$. Time to peak and peak response amplitude were calculated automatically within CIAT. The decay time constant was calculated manually in Excel 365 (Microsoft). $\tau$ was defined as the time point where the curve passed $<36.79 \%$ of the peak amplitude. Area under the curve (AUC) was calculated using the trapezoidal rule within Sigmaplot 12.0 (Systat Software). For some neurons, there was rundown in the calcium baseline over time due to photobleaching, leading to a negative AUC. This was left uncorrected.

$t$ tests were also performed in Sigmaplot 12.0. All $t$ tests involving pre-post trials were performed as paired $t$ tests. Before running paired $t$ tests, the normality of the data was tested (Shapiro-Wilk test); and if the data failed this test of normality $(p<0.05)$, a Wilcoxon signed-rank test was performed in lieu of a paired $t$ test. $t$ tests involving data from different neurons were performed as unpaired $t$ tests. If the data failed a test of normality (Shapiro-Wilk test), then a Mann-Whitney rank sum test was performed instead. For data involving more than two comparisons with one factor, one-way ANOVAS were performed. All one-way ANOVAs were run within SPSS (IBM). If the data failed a test of normality (Shapiro-Wilk test), a Kruskal-Wallis ANOVA on ranks was used. For the experiments described within Figure 10, where there were two factors with multiple groups, a two-way ANOVA was performed within Sigmaplot. Tukey's post hoc tests were used. The statistical test performed for each experiment is included in the figure legends. All bar graphs represent data as mean \pm SEM.

\section{Results}

\section{Resolving single AP-evoked calcium responses in lamina I neurons}

To investigate AP-evoked calcium responses, we performed simultaneous whole-cell patch-clamp recording and two-photon calcium $\left(\mathrm{Ca}^{2+}\right)$ imaging from lamina I neurons in acute 
A

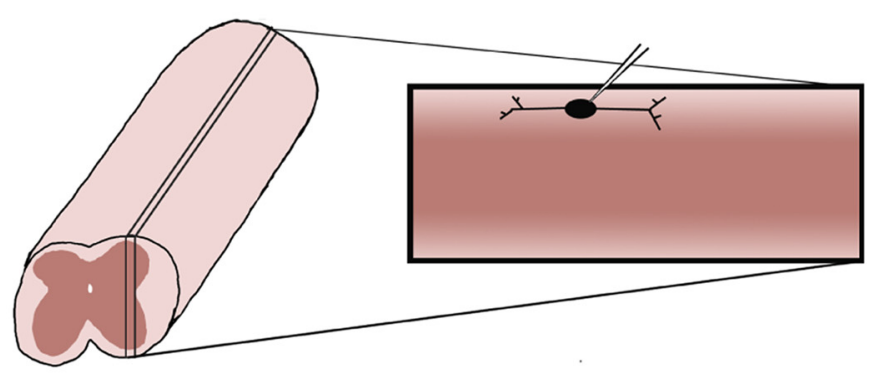

B

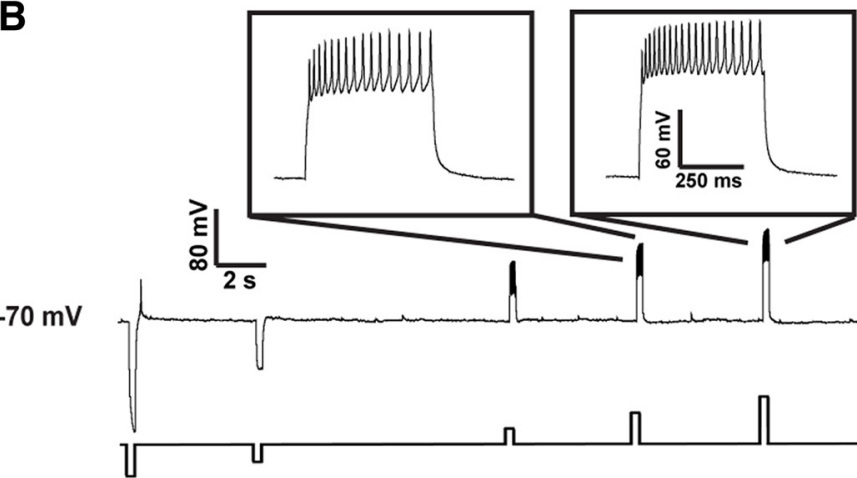

OGB-1

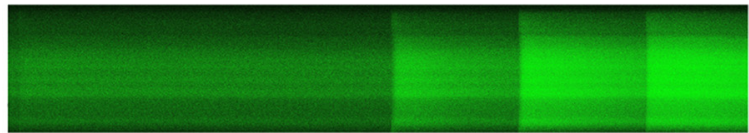

AF-594

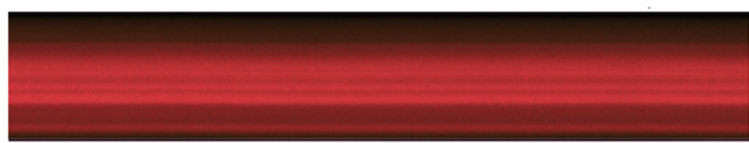

C

\section{Pre}

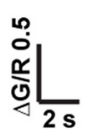

$\triangle G / R$

F

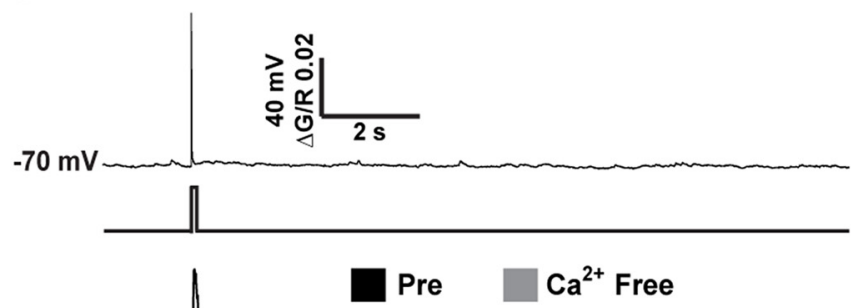

Why

$\Delta \mathrm{G} / \mathbf{R}$

RWy
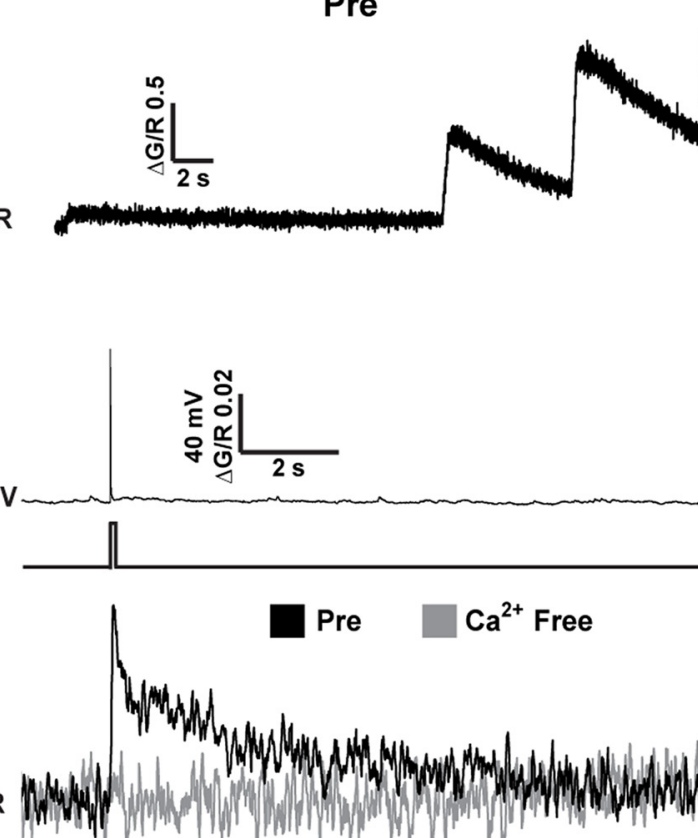
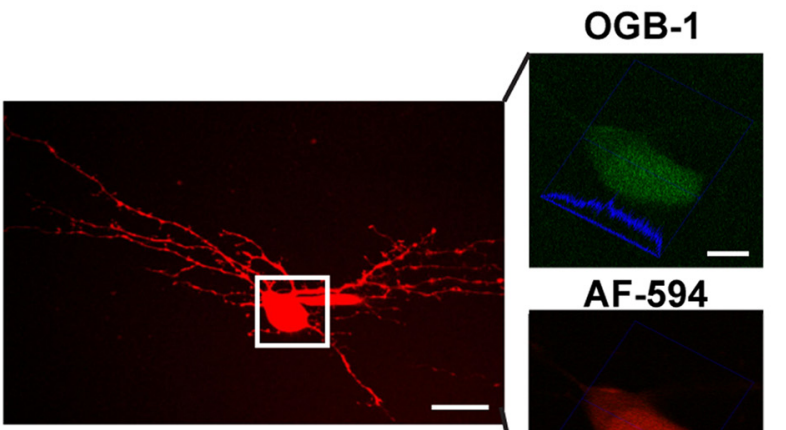

AF-594
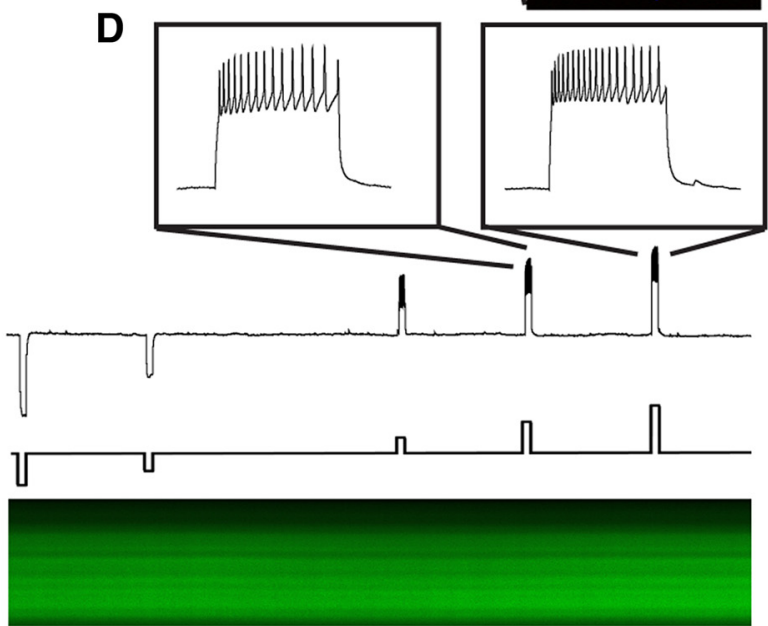

E

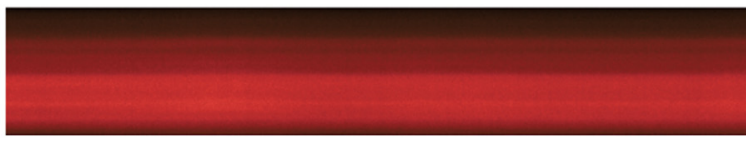

$\mathrm{Ca}^{2+}$ Free

G

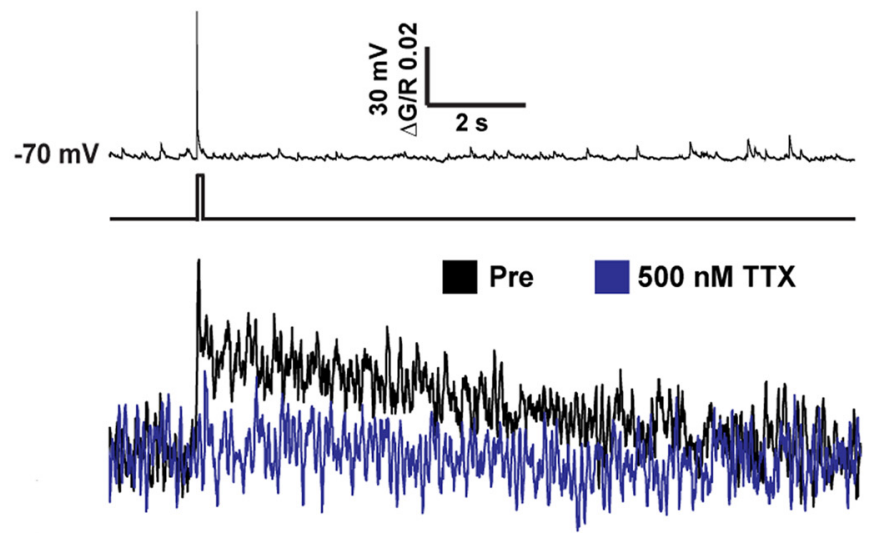

Figure 1. Resolving AP-evoked calcium responses in lamina I neurons. A, Schematic representing procedure for obtaining lamina I neuron recordings in spinal cord slices. Right, Flattened $z$ stack of a lamina I neuron. Scale bar, $20 \mu \mathrm{m}$. White box represents selection for inset. Inset, Frame scan depicting line scan selection through the soma, split by emission spectra into green and red channels corresponding to fluorescence from the calcium-sensitive dye OGB-1 and the structural dye AF-594, respectively. Inset, Scale bars, $5 \mu \mathrm{m}$. B, Top, Current-clamp trace of $500 \mathrm{~ms}$ steps $(-120 \mathrm{pA}, 50 \mathrm{pA}$ per step) and current injection trace. Bottom, Time-locked line scan image of 0GB-1 fluorescence and AF-594 fluorescence. C, Calculated $\Delta G / R$ response in the same neuron's soma. D, E, Perfusion of ACSF with no added calcium and $300 \mu \mathrm{m} \mathrm{EGTA}\left(\mathrm{Ca}^{2+}\right.$-free, gray) eliminated any AP-evoked changes in $\Delta \mathrm{G} / \mathrm{R}$. Calibration: Top, $y$ axis $=60 \mathrm{mV}, x$ 

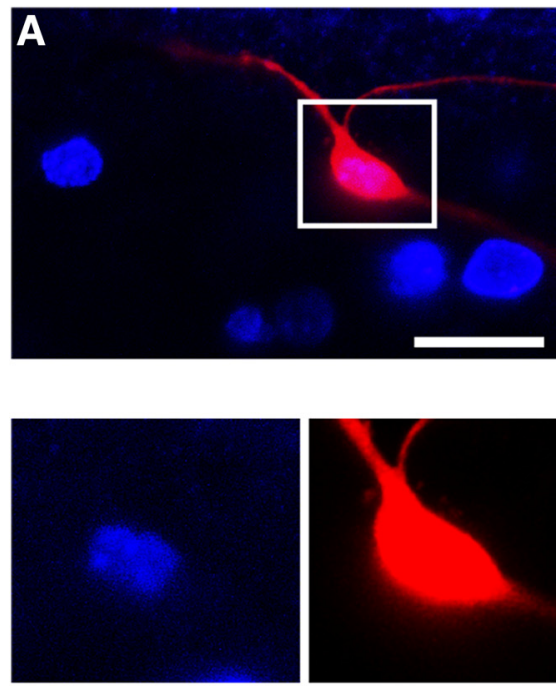

DAPI

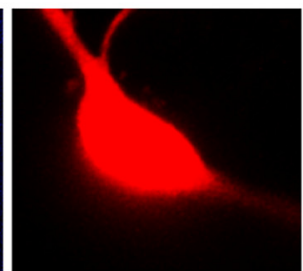

AF-594

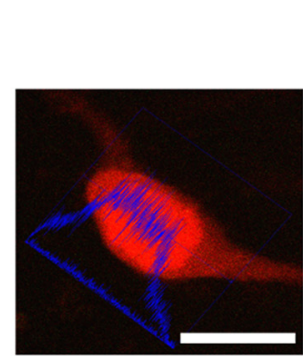

AF-594

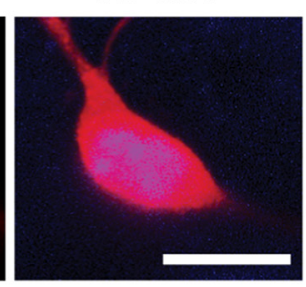

Overlay
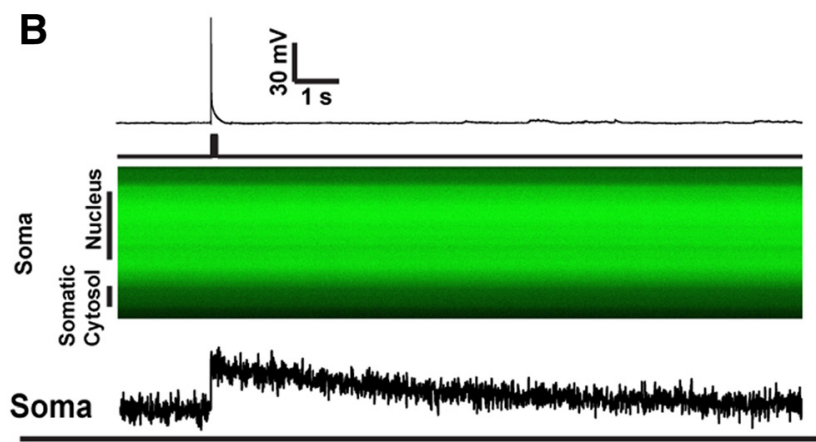

ํํㅇ

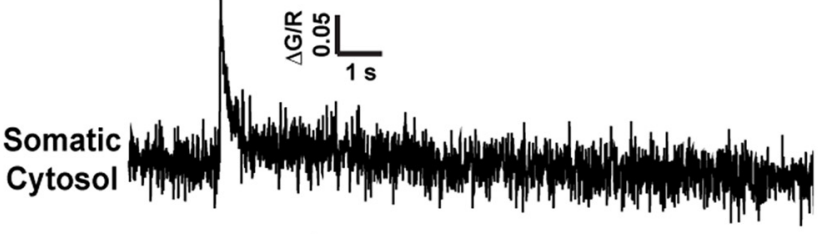

Nucleus

Figure 2. Somatic calcium responses are composed of cytosolic and nuclear components. $A$, Somatic line scans can be split into cytosolic and nuclear compartments based on differential AF594 fluorescence intensity. Postrecording addition of DAPI to the recording chamber confirmed that AF-594 sequesters to the nucleus producing a greater fluorescence intensity during recordings. Top right, AF-594 during recording. Top left and bottom, Flattened z-stack images after recording of AF-594 and DAPI nuclear stain. Scale bars: top left, $40 \mu$ m; top right and bottom right, $20 \mu \mathrm{m} . \boldsymbol{B}$, Top, Current-clamp trace, current injection, and OGB-1 fluorescence over time through the line scan selection in $A$. Calibration: $y$ axis $=30 \mathrm{mV}, x$ axis $=1 \mathrm{~s}$. Bottom, Calculated $\Delta \mathrm{G} / \mathrm{R}$ trace for the somatic line scan of the neuron in $\boldsymbol{A}$. Cytosolic and nuclear components were then extracted from the somatic line scan, revealing differences in compartmental calcium responses. Calibration: $y$ axis $=\Delta G / R$ 0.05, $x$ axis $=1 \mathrm{~s}$.

parasagittal slices of rat lumbar spinal cord (Hildebrand et al., 2014) (Fig. 1A). The calcium-sensitive indicator OGB-1 (110 $\mu \mathrm{M})$ and the calcium-insensitive, structural fluorophore Alexa Fluor 594 (AF-594; $35 \mu \mathrm{M}$ ) were dissolved in the intracellular recording solution and introduced into the neuron via the patch pipette. These fluorophores were simultaneously excited by a two-photon laser tuned to $800 \mathrm{~nm}$ and were split based on their emission spectra and sent to separate nondescanned photomultiplier tubes. Fluorescence data were acquired in line scan mode recorded at $133 \mathrm{~Hz}$. For each time point, the emission intensity of OGB-1 (G) was divided by that of AF-594 (R), and the difference in the ratio compared with a $2 \mathrm{~s}$ baseline $(\Delta \mathrm{G} / \mathrm{R})$ was calculated throughout each trial creating a continuous plot of instantaneous $\Delta G / R$ (Yasuda et al., 2003; Carter and Sabatini, 2004; Fekete et al., 2014) (e.g., Fig. 1C). AP firing was evoked by depolarizing the neuron with intracellular current injection. With prolonged depolarizations sufficient to induce trains of APs, we observed increases in $\Delta \mathrm{G} / \mathrm{R}$ in the neuronal soma that were graded by the stimulus amplitude (Fig. 1B,C). These depolarization-induced increases in $\Delta G / R$ were prevented by inhibiting entry of all extracellular calcium via perfusion of the slice with $\mathrm{Ca}^{2+}$-free ACSF containing the $\mathrm{Ca}^{2+}$ chelator EGTA, indicating that $\Delta \mathrm{G} / \mathrm{R}$ reliably reports on changes in intracellular $\mathrm{Ca}^{2+}$ concentration.

Single APs evoked by short ( $5 \mathrm{~ms}$ ), suprathreshold depolarizing current steps also evoked increases in $\Delta \mathrm{G} / \mathrm{R}$ (Fig. $1 F, G$ ). These increases were once again prevented by perfusing with

$\leftarrow$

axis $=2 \mathrm{~s}$; Inset, $y$ axis $=40 \mathrm{mV}, x$ axis $=250 \mathrm{~ms}$; Bottom, $y$ axis $=\Delta \mathrm{G} / \mathrm{R}$ 0.6, $x$ axis $=2 \mathrm{~s}$. $\boldsymbol{F}$, Smaller current steps ( $5 \mathrm{~ms}, 200 \mathrm{pA}$ ) elicited single APs and $\Delta \mathrm{G} / \mathrm{R}$ responses in the soma. Perfusion of ACSF with no added calcium and $300 \mu \mathrm{m}$ EGTA again eliminated all fluorescence changes ( $n=4$ neurons). $G$, Single AP-evoked $\Delta G / R$ responses in the soma were also ablated by the wash-in of $500 \mathrm{~nm}$ Tetrodotoxin (TTX) (dark blue, $n=7$ neurons). Calibrations: $\boldsymbol{C}, \boldsymbol{D}$, y axis $=40 \mathrm{mV}, \Delta \mathrm{G} / \mathrm{R} 0.02, x$ axis $=2 \mathrm{~s}$. C, $\boldsymbol{D}$, Calcium imaging data presented as mean.
$\mathrm{Ca}^{2+}$-free ACSF plus EGTA (Fig. $1 F$ ). Blocking AP generation by perfusing with ACSF containing TTX also prevented current steps from increasing $\Delta \mathrm{G} / \mathrm{R}$ (Fig. $1 G$ ). Together, these findings indicate the ability to resolve increases in intracellular $\mathrm{Ca}^{2+}$ concentration evoked by single APs. Using this approach, we studied $\mathrm{Ca}^{2+}$ responses in a total of 212 neurons in lamina I of the dorsal horn.

\section{Single AP-evoked calcium responses in subcellular} compartments of lamina I neurons

Single APs resulted in increases in $\Delta G / R$ in the soma of 210 of 212 recorded lamina I neurons. With line scans across the entire neuronal soma, encompassing both the cytosol and nucleus, we observed a rapid onset rise in $\Delta \mathrm{G} / \mathrm{R}$ in the soma that peaked within 150-200 ms. Following the peak, the decay in $\Delta G / R$ appeared as an initial fast decay phase of $\sim 30 \%-40 \%$ from peak $\Delta \mathrm{G} / \mathrm{R}$ within the first $200 \mathrm{~ms}$, followed by a slower secondary decay to baseline $\Delta \mathrm{G} / \mathrm{R}$ over the next $10 \mathrm{~s}$ (Fig. $1 F, G$ ). Separating the line scan into ROIs aligning to the cytosol and nucleus revealed that the amplitude and the time course of the cytosolic $\Delta \mathrm{G} / \mathrm{R}$ responses were markedly different from those in the nucleus (Fig. 2). Therefore, in all subsequent experiments, we separated nuclear from cytosolic compartments of the somatic $\Delta \mathrm{G} / \mathrm{R}$.

Whether APs generated in the soma of lamina I neurons affect intracellular $\mathrm{Ca}^{2+}$ concentration in the dendrites is currently unknown. Therefore, for each recorded neuron, we also measured $\Delta \mathrm{G} / \mathrm{R}$ in a primary dendrite $20-60 \mu \mathrm{m}$ from the soma (Fig. $3 A, B)$. We found that single APs produced an increase in dendritic $\Delta \mathrm{G} / \mathrm{R}$ in all of the 210 neurons with a somatic $\mathrm{Ca}^{2+}$ response. Thus, single APs not only cause a rise in intracellular $\mathrm{Ca}^{2+}$ in somatic cytosol and nucleus of lamina I dorsal horn neurons, but also within their dendrites.

Next, we compared $\Delta \mathrm{G} / \mathrm{R}$ responses between these three subcellular compartments. For each neuron, in each compartment, we computed the peak $\Delta \mathrm{G} / \mathrm{R}$, the time to peak from current 

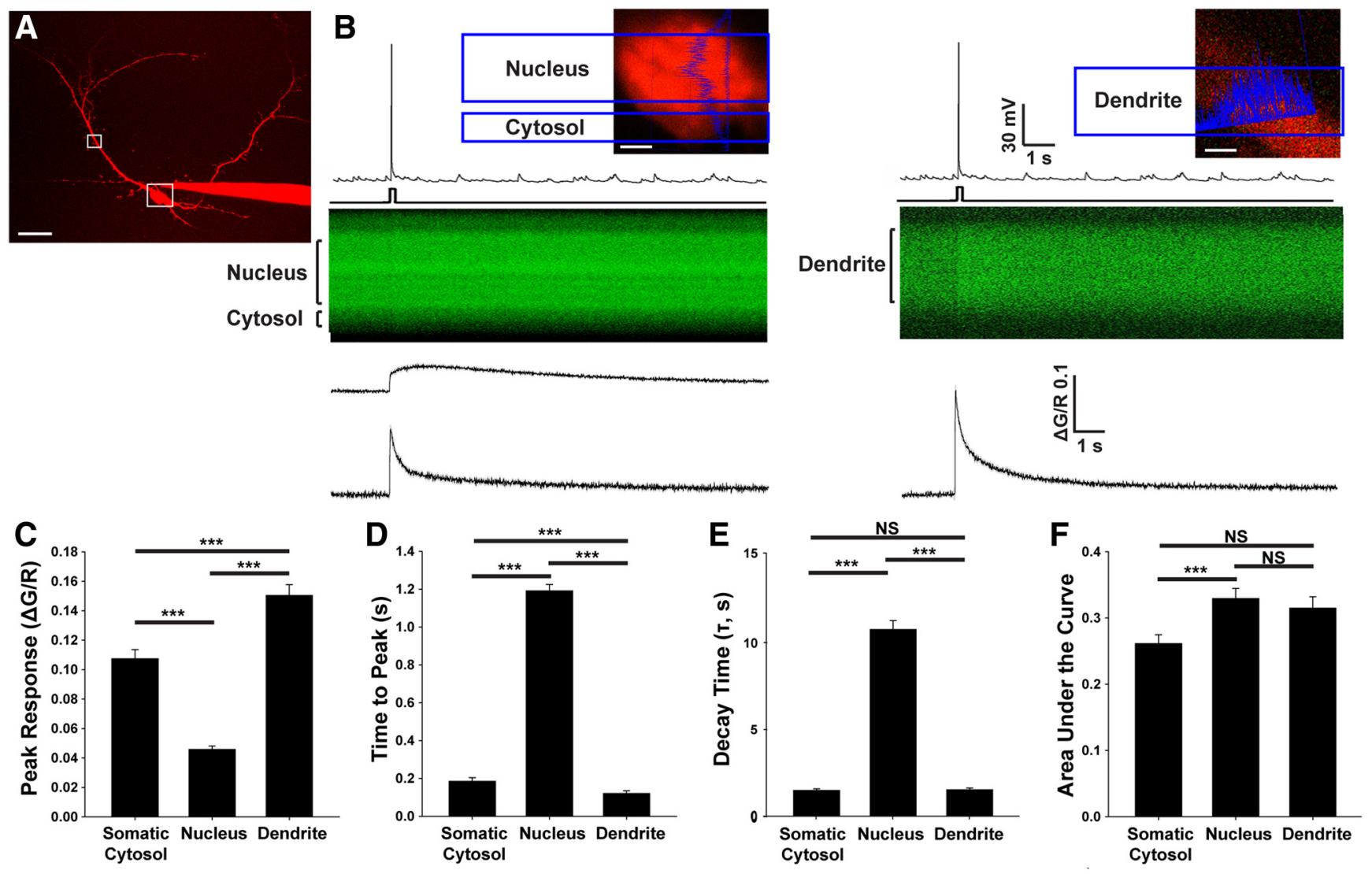

G

Pre
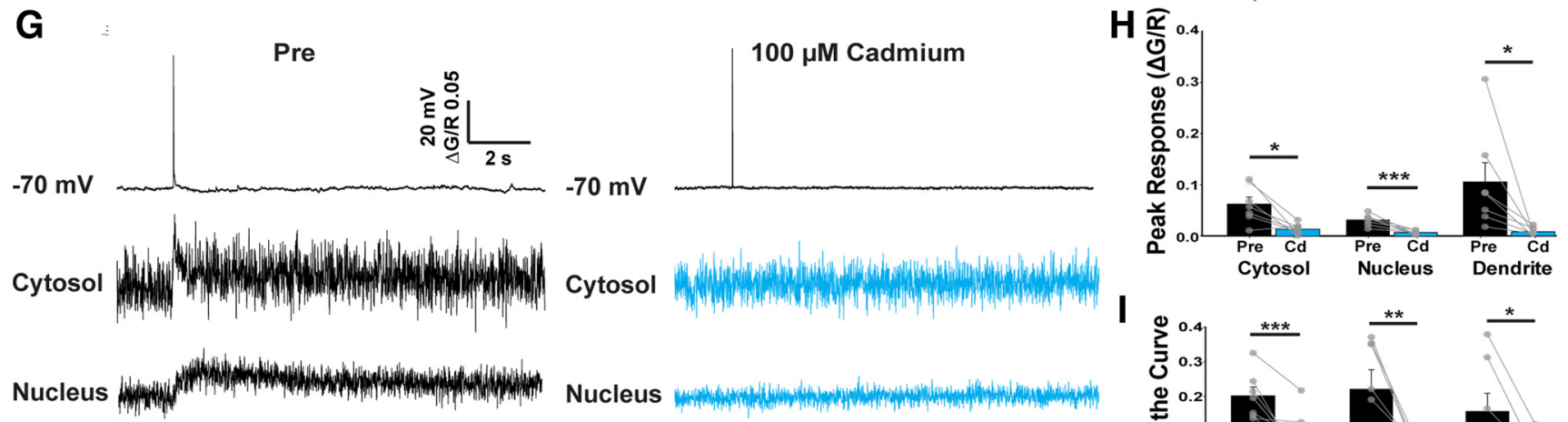

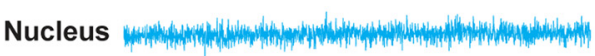

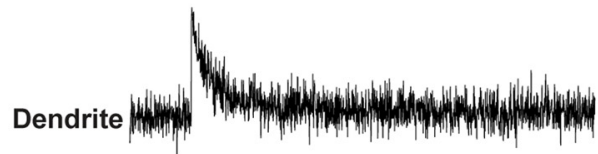

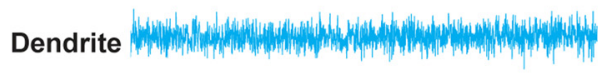

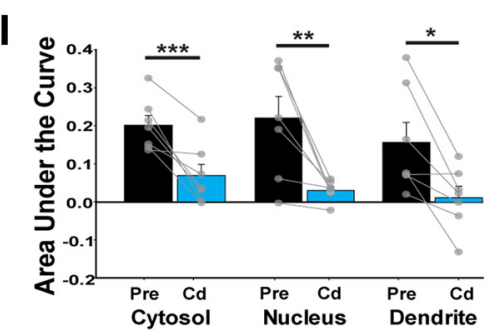

Figure 3. Single AP-evoked calcium responses in subcellular compartments of lamina I neurons. $\boldsymbol{A}$, Two-photon flattened $z$-stack image of an example lamina I neuron (Scale bar, $30 \mu \mathrm{m}$ ), along with line scan selections. Scale bars: soma, $5 \mu \mathrm{m}$; dendrite, $0.5 \mu \mathrm{m}$. B. Current-clamp traces, current injection traces, and example line scans of 0GB-1 for each compartment of this neuron. Bottom, $\Delta \mathrm{G} / \mathrm{R}$ mean traces for each compartment (for all compartments, $n=210$ neurons). Calibration: Top, $y$ axis $=25 \mathrm{mV}, x$ axis $=1 \mathrm{~s} ;$ Bottom, $y$ axis $=\Delta \mathrm{G} / \mathrm{R} 0.1, x$ axis $=1 \mathrm{~s}$. $C$, Mean peak response compared between somatic cytosol, nucleus, and dendrites ( $n=210$ neurons from 172 mice, Kruskal-Wallis one-way ANOVA, $p<0.001)$. Post hoc multiple comparisons by Tukey's test (cytosol vs nucleus, $p<0.001$; cytosol vs dendrite, $p=0.006$; nucleus vs dendrite, $p<0.001$ ). $\boldsymbol{D}$, Mean time to peak compared between somatic cytosol, nucleus, and dendrites ( $n=210$ neurons from 172 mice, Kruskal-Wallis one-way ANOVA, $p<0.001$ ). Post hoc multiple comparisons by Tukey's test (cytosol vs nucleus, $p<0.001 ;$ cytosol vs dendrite, $p=0.002$; nucleus vs dendrite, $p<0.001)$. $\boldsymbol{E}$, Mean decay time compared between somatic cytosol, nucleus, and dendrites $(n=210$ neurons from 172 mice, Kruskal-Wallis one-way ANOVA, $p<0.001$ ). Post hoc multiple comparisons by Tukey's test (cytosol vs nucleus, $p<0.001$; cytosol vs dendrite, $p=1.000$; nucleus vs dendrite, $p<0.001$ ). $\boldsymbol{F}$, Mean AUC compared between somatic cytosol, nucleus, and dendrites ( $n=210$ neurons from 172 mice, Kruskal-Wallis one-way ANOVA, $p=0.001$ ). Post hoc multiple comparisons by Tukey's test (cytosol vs nucleus, $p=0.002 ;$ cytosol vs dendrite, $p=0.120$; nucleus vs dendrite, $p=0.656)$. $\mathbf{G}$, Mean $\Delta \mathrm{G} / \mathrm{R}$ responses to single APs before (black) and after (light blue) perfusion of $100 \mu \mathrm{m}$ cadmium (Cd, $n=7$ neurons). $\boldsymbol{H}$, Mean peak response before and after perfusion of $\mathrm{Cd}(n=7$ neurons, paired $t$ tests were performed for each compartment: cytosol $p=0.014$, nucleus $p=0.002$, dendrite $p=0.016$ ). $I$, Mean AUC before and after perfusion of $\mathrm{Cd}(n=7$ neurons, paired $t$ tests were performed for each compartment: cytosol $p=0.003$, nucleus $p=0.01$, dendrite $p=0.012$ ). Calibrations: $\boldsymbol{B}, \boldsymbol{C}, y$ axis $=20 \mathrm{mV}, \Delta \mathrm{G} / \mathrm{R} 0.05, x$ axis $=2$ s. Data are mean \pm SEM. ${ }^{*} p<0.05$. ${ }^{* *} p<0.01$. ${ }^{* *} p<0.005$. Where indicated in all subsequent figures, NS $=$ not statistically significant, $p>0.05$.

injection, the decay rate following peak response, and the area under the $\Delta \mathrm{G} / \mathrm{R}$ curve (AUC). To determine the peak $\Delta \mathrm{G} / \mathrm{R}$, we measured the maximum instantaneous $\Delta \mathrm{G} / \mathrm{R}$ reached after the AP for each neuron, in each compartment. We found that mean peak $\Delta \mathrm{G} / \mathrm{R}$ was significantly different in each compartment (one-way ANOVA $p<0.001$; Fig. $3 C$ ), and peak $\Delta \mathrm{G} / \mathrm{R}$ was greatest in the dendritic compartment $(0.151 \pm 0.007)$, and lowest within the nuclear compartment $(0.046 \pm 0.002)$. 

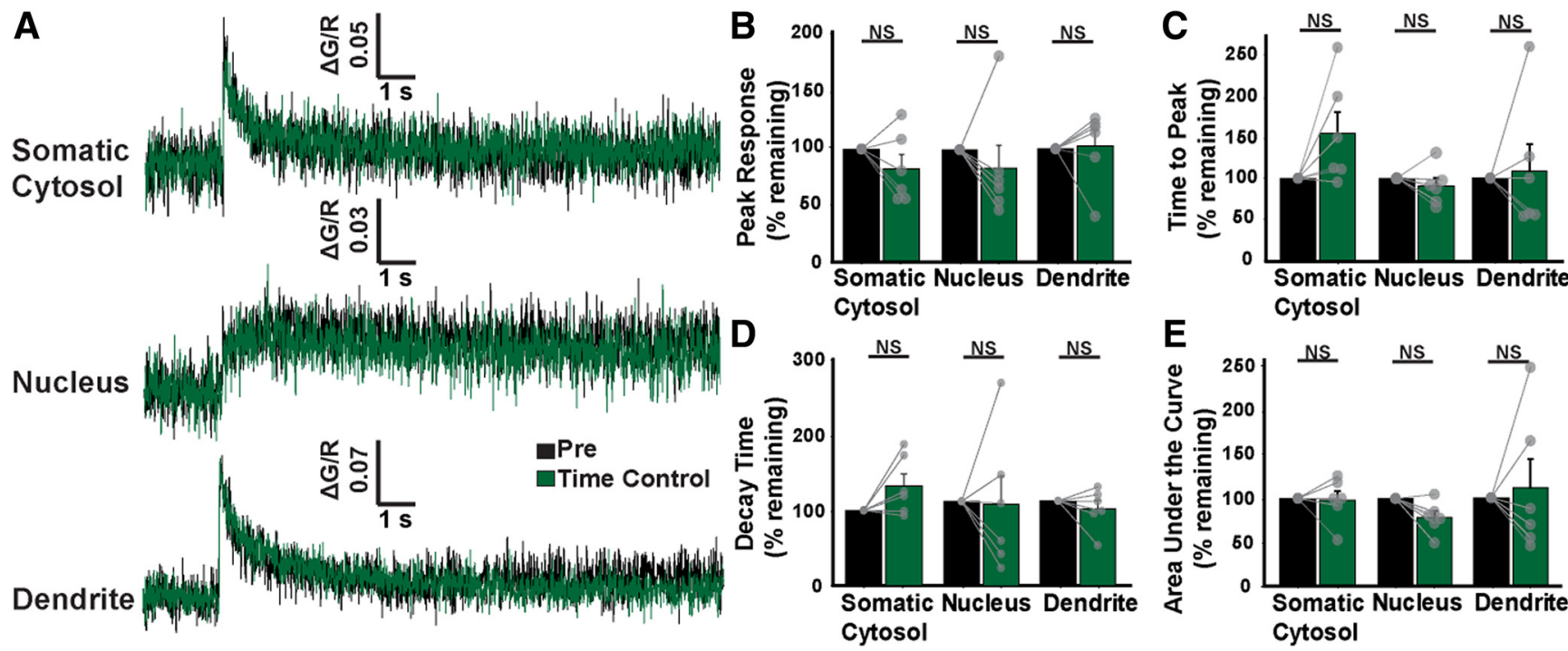

Figure 4. AP-evoked calcium responses are consistent over experimental duration. $\boldsymbol{A}$, Mean calcium responses before (black) and after (green) experimental duration (20 min; $n=6$ neurons). Calibration: Top, $y$ axis $=\Delta G / R$ 0.06, $x$ axis $=1 \mathrm{~s}$; Middle, $y$ axis $=\Delta G / R$ 0.03, $x$ axis $=1 \mathrm{~s} ;$ Bottom, $y$ axis $=\Delta G / R$ 0.08, $x$ axis $=1 \mathrm{~s}$. $\boldsymbol{B}-\boldsymbol{E}$, Quantification of calcium responses before (black) and after (green) $20 \mathrm{~min}$ in all three subcellular compartments. $\boldsymbol{B}$, Peak response. Paired $t$ test for somatic cytosol $p=0.476$, nucleus $p=0.438$, dendrite $p=0.548$. $\boldsymbol{C}$, Time to peak. Paired $t$ test for somatic cytosol $p=0.563$, nucleus $p=0.233$, dendrite $p=0.783$. $D$, Decay time constant $(\tau)$. Paired $t$ test for somatic cytosol $p=0.073$, nucleus $p=0.550$, dendrite $p=0.313$. $E$, AUC. Paired $t$ test for somatic cytosol $p=0.840$, nucleus $p=0.064$, dendrite $p=0.341$.

In the somatic cytosol and the dendrites, the increase in $\Delta \mathrm{G} / \mathrm{R}$ appeared as a single rising phase, whereas in the nucleus the rise time consisted of two phases. As a result, the time to peak of the $\Delta \mathrm{G} / \mathrm{R}$ response varied significantly across the three subcellular compartments (one-way ANOVA, $p<0.001$; Fig. $3 D)$. In the nucleus, the time to $\Delta \mathrm{G} / \mathrm{R}$ peak was much longer compared with the somatic cytosol or dendrites $(1.19 \pm 0.03 \mathrm{~s}$ in the nucleus vs $0.185 \pm 0.02$ and $0.121 \pm 0.01 \mathrm{~s}$ in the somatic cytosol and dendrites, respectively). The slow secondary phase of the nuclear response indicates that $\Delta \mathrm{G} / \mathrm{R}$ was continuing to increase in this compartment, even when $\Delta \mathrm{G} / \mathrm{R}$ had fallen substantially in the other two compartments, and long after the neuronal membrane potential had returned to the resting level.

We next examined the decay of $\Delta \mathrm{G} / \mathrm{R}$ by calculating the value of the decay time constant $(\tau)$ in each subcellular compartment, for each neuron. As with other measures, nuclear $\Delta \mathrm{G} / \mathrm{R}$ responses had widely divergent decay properties compared with the somatic cytosol and dendrites. In response to a single AP, the nuclear $\Delta \mathrm{G} / \mathrm{R}$ response had a mean decay time constant of $10.74 \pm 0.52 \mathrm{~s}$, compared with $1.50 \pm 0.09$ and $1.52 \pm 0.11 \mathrm{~s}$ for the somatic cytosolic and dendritic compartments, respectively (one-way ANOVA, $p<0.001$; Fig. $3 E$ ).

To measure the overall integrated $\mathrm{Ca}^{2+}$ response to AP firing in each subcellular compartment, we calculated the AUC for each $\Delta G / R$ response. When comparing responses between subcellular compartments (Fig. $3 F$ ), we found that the AUC of the somatic cytosol was significantly less than that in the nucleus (one-way ANOVA, $p=0.001$, post hoc Tukey $t$ test, $p=0.002$ ). This indicates that, despite the decreased peak $\Delta \mathrm{G} / \mathrm{R}$ response in the nuclear compartment, the much slower decay rate within the nucleus leads to an overall larger integrated $\mathrm{Ca}^{2+}$ response due to AP firing.

We determined the stability of calcium responses over the recording period and found that $\Delta \mathrm{G} / \mathrm{R}$ responses were stable during the total recording period, with no significant change in $\Delta \mathrm{G} /$ $\mathrm{R}$ responses before and after a $20 \mathrm{~min}$ time control $(n=6$ neurons; Fig. 4). Next, we considered the possibility that evoking APs through current injection may not accurately reflect $\Delta \mathrm{G} / \mathrm{R}$ responses to spontaneously occurring APs in lamina I neurons.
However, we found that, as with APs evoked by current injection, spontaneous APs similarly increased $\Delta \mathrm{G} / \mathrm{R}$ in the somatic cytosol, nucleus, and dendrites of lamina I neurons ( $n=6$ neurons; Fig. 5), and in none of these compartments was there a statistically significant difference in peak $\Delta \mathrm{G} / \mathrm{R}$ response between spontaneous and evoked APs. In addition, in the absence of an AP, current injection failed to elicit an increase in $\Delta \mathrm{G} / \mathrm{R}$ (Fig. 6). Thus, the $\Delta \mathrm{G} / \mathrm{R}$ responses evoked by current injection appear to recapitulate the responses produced by spontaneously occurring APs in these neurons.

The $\Delta \mathrm{G} / \mathrm{R}$ responses in the soma were prevented by TTX and by removing extracellular calcium (Figs. $1 G, 6$ ), suggesting that influx of $\mathrm{Ca}^{2+}$ through VGCCs is necessary for AP-induced $\Delta \mathrm{G} / \mathrm{R}$ responses. To determine whether the $\Delta \mathrm{G} / \mathrm{R}$ responses in the three subcellular compartments require VGCCs, we examined the effect of bath application of the VGCC blocker cadmium (Fig. 3G). During perfusion of cadmium (100 $\mu \mathrm{m})$, AP-evoked $\Delta \mathrm{G} / \mathrm{R}$ responses were prevented in each compartment (peak $\Delta \mathrm{G} / \mathrm{R}$ decreased from $0.129 \pm 0.025$ to $0.014 \pm 0.007$ in somatic cytosol, $0.045 \pm 0.007$ to $0.008 \pm 0.003$ in nucleus, and $0.186 \pm 0.031$ to $0.008 \pm 0.003$ in dendrites; $n=7$ neurons). Therefore, we conclude that $\mathrm{Ca}^{2+}$ influx through VGCCs is necessary to evoke these $\Delta \mathrm{G} /$ $\mathrm{R}$ responses in the somatic cytosol, nucleus, and dendrites.

Overall, we found the most divergent kinetics within the nuclear compartment, which despite having the smallest peak $\Delta \mathrm{G} /$ $\mathrm{R}$ response, also exhibited the slowest rise and decay of the $\Delta \mathrm{G} / \mathrm{R}$ response compared with any other subcellular compartment. This indicates a slow and persistent elevation in nuclear $\mathrm{Ca}^{2+}$ concentration in response to AP firing, a phenomenon we observed in all lamina I neurons. These findings also indicate that, despite differences in the kinetics of the $\Delta \mathrm{G} / \mathrm{R}$ responses induced by APs, responses in all subcellular compartments are dependent on $\mathrm{Ca}^{2+}$ influx via VGCCs for response initiation.

Calcium-induced calcium release (CICR) contributes to somatic calcium responses in lamina I neurons CICR from ryanodine-sensitive intracellular stores has been shown to contribute to and elongate AP-induced calcium 

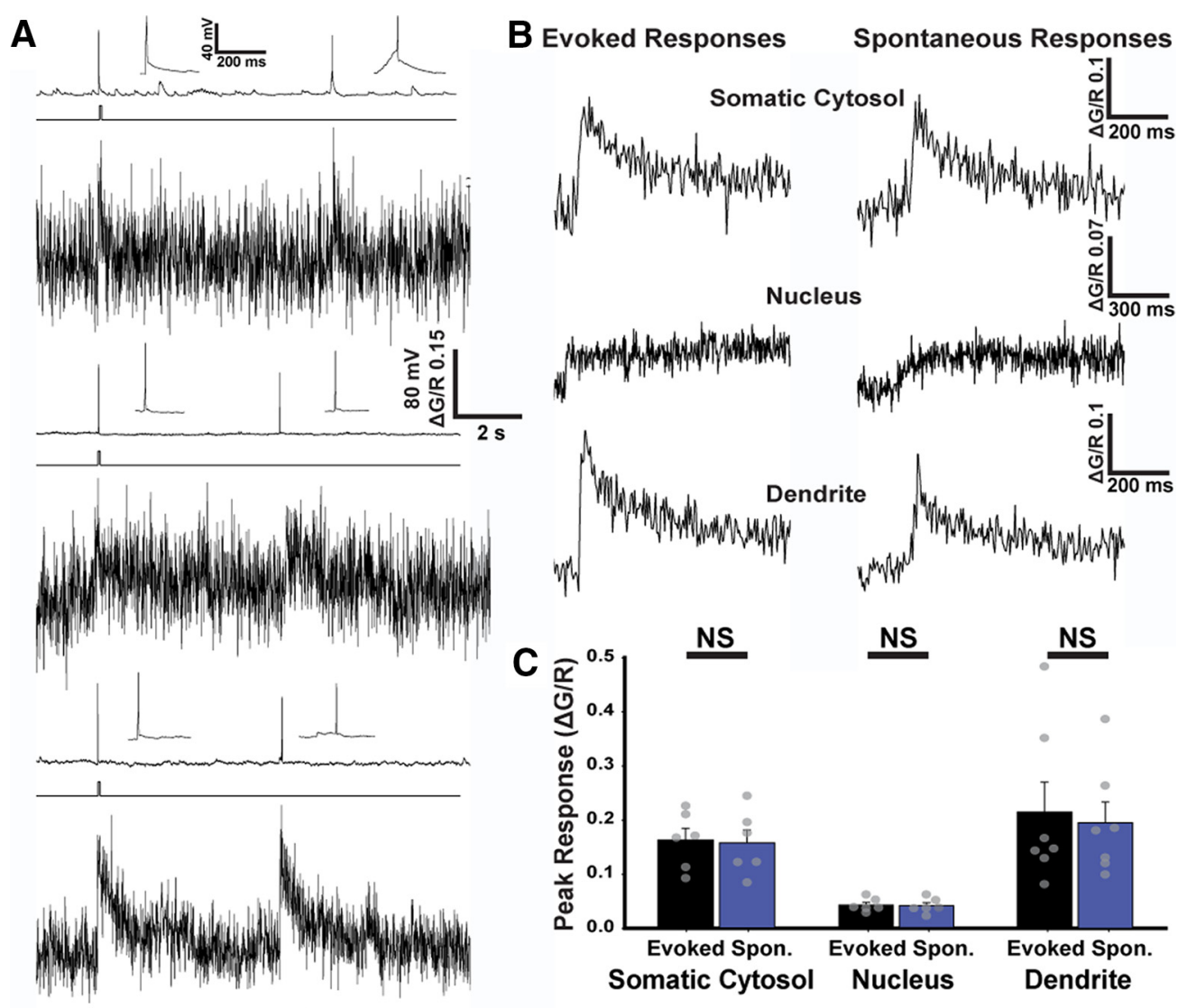

Figure 5. Comparison of evoked and spontaneous calcium responses. $A$, Example current-clamp traces, current injection traces, and calcium responses in dendrites. Single APs were evoked as indicated by current injection; spontaneous APs were recorded and measured as well. Calibration: $y$ axis $=80 \mathrm{mV}, \Delta \mathrm{G} / \mathrm{R} 0.15, x$ axis $=2 \mathrm{~s}$. Calibration: Inset, $y$ axis $=40 \mathrm{mV}, x$ axis $=200 \mathrm{~ms}$. $B$, Mean calcium responses in cytosolic, nuclear, and dendritic compartments from evoked APs (left) and spontaneous APs (right) ( $n=6$ neurons). Calibration: Top, $y$ axis $=\Delta \mathrm{G} / \mathrm{R}$ 0.1, $x$ axis $=200 \mathrm{~ms}$; Middle, $y$ axis $=\Delta G / R$ 0.07, $x$ axis $=300 \mathrm{~ms}$; Bottom, $y$ axis $=\Delta G / R$ 0.1, $x$ axis $=200 \mathrm{~ms}$. C, Quantification of peak response between evoked and spontaneous APs. Paired $t$ test for somatic cytosol $p=0.584$, nucleus $p=0.782$, dendrite $p=0.393$. Data are mean \pm SEM.

responses in other types of neurons (Sandler and Barbara, 1999; Sjöstrom and Nelson, 2002). As ryanodine receptors are known to be expressed in the superficial spinal cord (Kyrozis et al., 1996; Cheng et al., 2010; Naka et al., 2013), we tested whether ryanodine receptor-mediated CICR contributes to $\Delta \mathrm{G} / \mathrm{R}$ responses in lamina I neurons. Ryanodine $(100 \mu \mathrm{M})$, which locks the receptors in a low conductance state thereby preventing CICR (Meissner, 2017), was applied by bath perfusion (Fig. 7; $n=7$ neurons). Addition of ryanodine had no effect on time to peak or on decay time in any of the compartments. However, we found that, while ryanodine had no effect on the peak $\Delta \mathrm{G} / \mathrm{R}$ responses in somatic cytosol or dendrites, it significantly reduced peak responses in the nucleus (paired $t$ test, $p=0.031$; Fig. $7 A, B$ ). In addition, ryanodine significantly decreased the AUC of both nuclear and cytosolic compartments (paired $t$ tests, $p=0.013$ and $p=0.016$, respectively), with no effect in the dendritic compartment (paired $t$ test, $p=0.606$; Fig. $7 E$ ). Together, these data demonstrate that ryanodine receptor-mediated CICR boosts lamina I nuclear and somatic cytosolic $\mathrm{Ca}^{2+}$ responses evoked by AP firing.

Lamina I calcium responses exhibit slower rise and decay kinetics compared with CA1 pyramidal neurons

While investigating AP-evoked $\Delta \mathrm{G} / \mathrm{R}$ responses in lamina I neurons, we noted two characteristics that appeared to differentiate these neurons from others reported in the literature. First, the decay time of the AP-induced $\Delta \mathrm{G} / \mathrm{R}$ responses in lamina $\mathrm{I}$ neurons (Fig. 3) appeared slower than in experiments in other neuronal populations (Sandler and Barbara, 1999; Rozsa et al., 2004). Second, the nuclear $\Delta \mathrm{G} / \mathrm{R}$ responses in lamina I neurons had two distinct rise phases, whereas the somatic cytosolic and dendritic compartments had a monophasic rise in $\Delta \mathrm{G} / \mathrm{R}$. To understand whether these differences were due to experimental conditions or inherent to lamina I neuron physiology, we directly compared $\Delta \mathrm{G} / \mathrm{R}$ responses with single AP firing between lamina I and hippocampal CA1 pyramidal neurons.

Lamina I neuron $\Delta \mathrm{G} / \mathrm{R}$ responses were much slower to peak within the nuclear compartment, indicative of the secondary slow rise phase being unique to lamina I neurons ( $t$ test, $p<$ $0.001, n=8$ neurons) (Fig. $8 D$ ). Similarly, the decay time constant in all compartments was larger in lamina I neurons (somatic cytosol $p=0.035$, nucleus $p=0.005$, dendrite $p=0.01$ ) (Fig. $8 E)$. Therefore, hippocampal CA1 pyramidal neuron responses were in general faster to rise and faster to decay, with no significant difference in AUC compared with lamina I neurons.

To determine whether these differences in $\Delta \mathrm{G} / \mathrm{R}$ response were due to differences in AP characteristics, we compared AP attributes between the cell types. Although hippocampal CA1 neuron APs had a slightly higher mean amplitude $(p=0.05, n=8$ neurons), no differences in rise time or decay time constants were found, and thus could not account for the differences in time to peak or decay kinetics observed in $\Delta \mathrm{G} / \mathrm{R}$ responses between these cell types (Fig. 8G-J). Together, these data indicate that AP-evoked $\Delta \mathrm{G} / \mathrm{R}$ responses are divergent between these two neuronal populations, and especially within the nuclear 
A

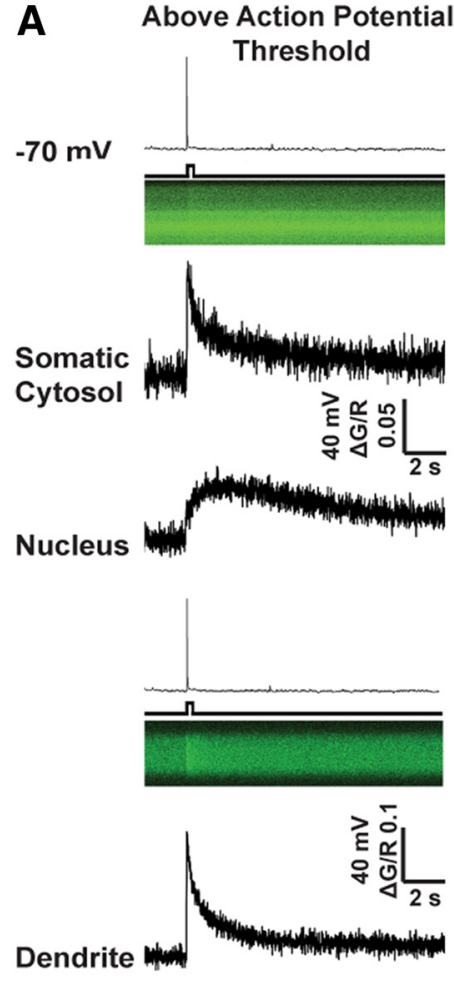

Below Action Potential B
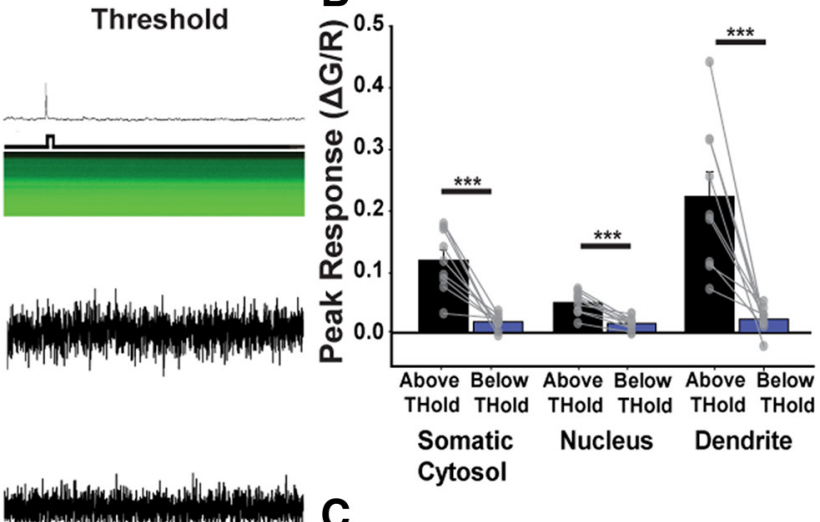

C
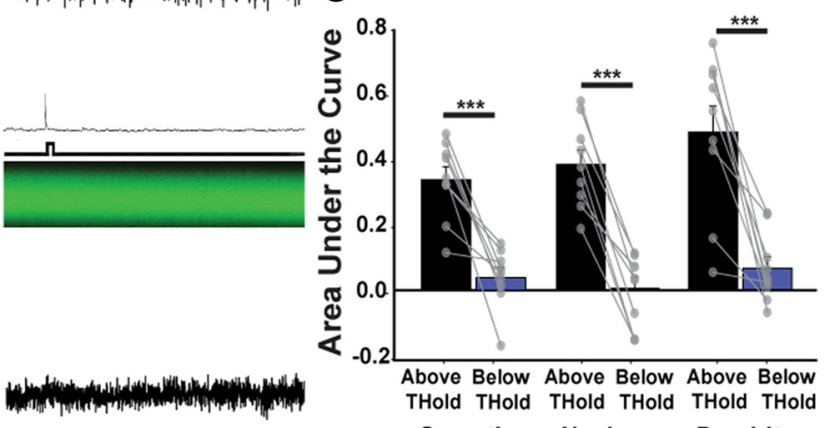

Somatic

Nucleus Dendrite

D

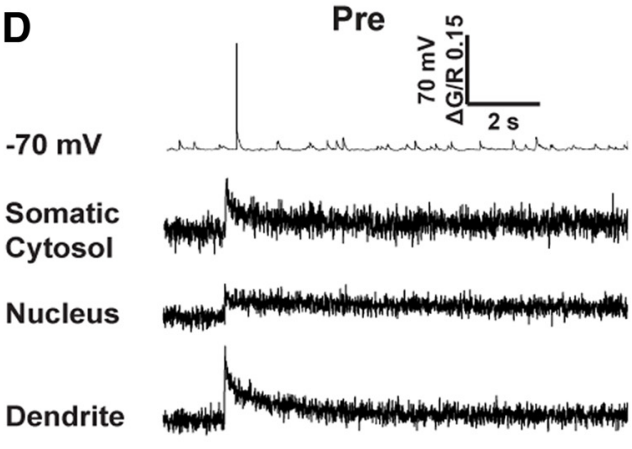

500 nM Tetrodotoxin

$-70 \mathrm{mV}$

Somatic

Cytosol

Nucleus

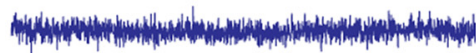

Dendrite
E

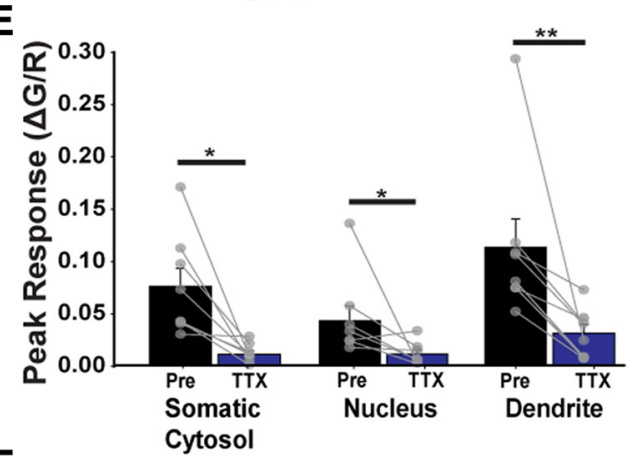

$\mathbf{F}$

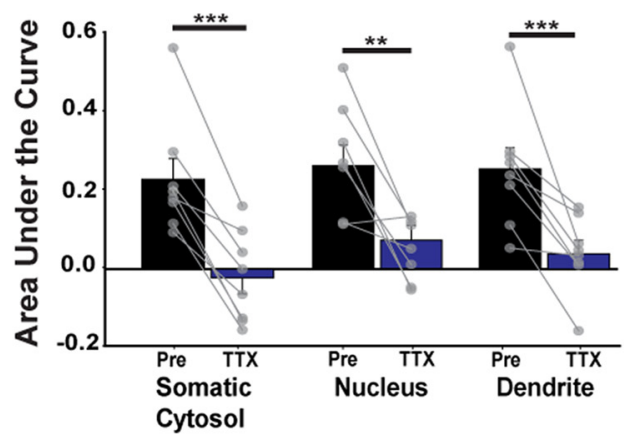

Figure 6. APs are required to elicit calcium responses. $\boldsymbol{A}$, The $5 \mathrm{~ms}$ current injections were evoked at $180 \mathrm{pA}$ ( $50 \%$ failure rate), and calcium responses were grouped by successful and failed APs. Mean calcium responses for each compartment above and below AP are displayed ( $n=9$ neurons, mean of 2-7 trials per neuron). Calibration: Top, $y$ axis $=40 \mathrm{mV}, \Delta \mathrm{G} / \mathrm{R} 0.05, x$ axis $=2 \mathrm{~s}$; Bottom, $y$ axis $=40 \mathrm{mV}, \Delta \mathrm{G} / \mathrm{R} 0.1, x$ axis $=2 \mathrm{~s}$. B. Peak calcium response above and below AP threshold (THold) in somatic cytosol, nucleus, and dendrites. Paired $t$ test for compartments: somatic cytosol $p<0.001$, nucleus $p<0.001$, dendrite $p=0.002$. C, AUC above and below AP threshold. Paired $t$ test for compartments: somatic cytosol $p<0.001$, nucleus $p$ $<0.001$, dendrite $p<0.001$. D, Mean $\Delta G / R$ responses in each subcellular compartment before (black) and after (dark blue) the wash-in of TTX (500 nm; $n=7 \mathrm{neurons}$ for somatic cytosol and nucleus, and 8 neurons for dendrites). Calibration: $y$ axis $=70 \mathrm{mV}, \Delta G / R$ 0.15, $x$ axis $=2 \mathrm{~s}$. $E$, Peak calcium response before and after wash-in of TTX. Paired $t$ test for compartments: somatic cytosol $p=0.014$, nucleus $p=0.047$, dendrite $p=0.008$. $F$, AUC before and after wash-in of TTX. Paired $t$ test for compartments: somatic cytosol $p<0.001$, nucleus $p=0.007$, dendrite $p=0.004 . \boldsymbol{B}, \boldsymbol{C}, \boldsymbol{E}, \boldsymbol{F}$, Data are mean \pm SEM. ${ }^{*} p<0.05$. ${ }^{* *} p<0.01 .{ }^{* * *} p<0.005$. 
A

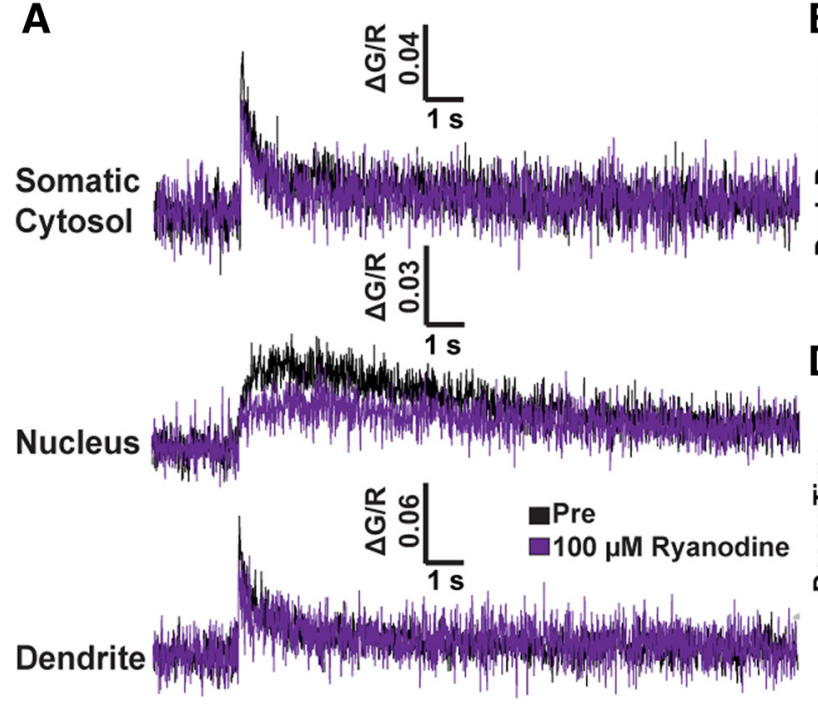

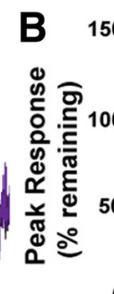

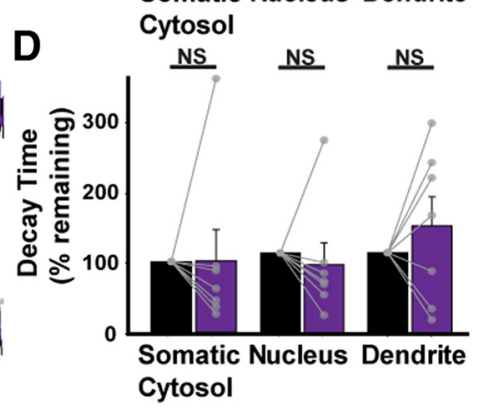

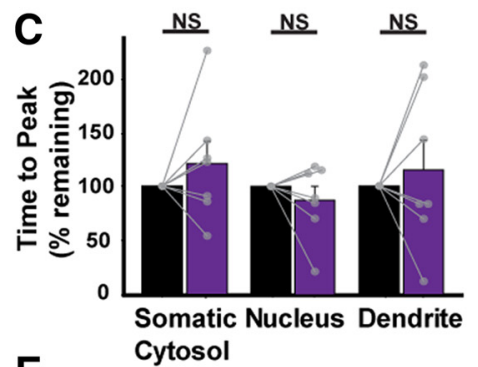

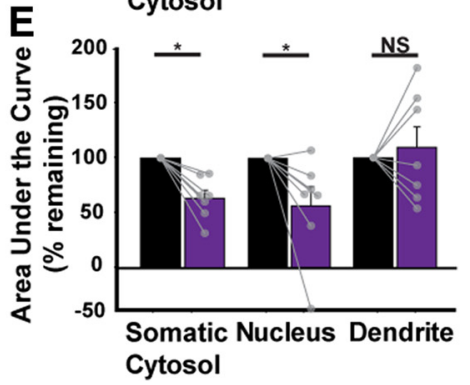

Figure 7. CICR contributes to somatic calcium responses in lamina I neurons. $A$, Mean $\Delta G / R$ responses, before (black) and after (purple) perfusion of ryanodine (100 $\mu \mathrm{m} ; n=7$ neurons). Calibration: Top, $y$ axis $=\Delta \mathrm{G} / \mathrm{R} 0.1, x$ axis $=1 \mathrm{~s}$; Middle, $y$ axis $=\Delta \mathrm{G} / \mathrm{R} 0.05, x$ axis $=1 \mathrm{~s}$; Bottom, $y$ axis $=\Delta \mathrm{G} / \mathrm{R} 0.08, x$ axis $=1 \mathrm{~s}$. $\boldsymbol{B}-\boldsymbol{E}$, Quantification of $\Delta \mathrm{G} / \mathrm{R}$ responses before (black) and after (purple) perfusion of ryanodine in all three subcellular compartments ( $n=7$ neurons). $\boldsymbol{B}$, Peak response. Paired $t$ tests were performed for each compartment: cytosol $p=0.469$, nucleus $p=0.031$, dendrite $p=0.164$. C, Time to peak. Paired $t$ tests were performed for each compartment: cytosol $p=0.627$, nucleus $p=0.354$, dendrite $p=0.938$. $\boldsymbol{D}$, Decay time constant $(\tau)$. Paired $t$ tests were performed for each compartment: cytosol $p=0.447$, nucleus $p=0.318$, dendrite $p=0.687$. $E$, AUC. Paired $t$ tests were performed for each compartment: cytosol $p=0.016$, nucleus $p=0.013$, dendrite $p=0.606$. $\boldsymbol{B}-\boldsymbol{E}$, Data are normalized mean \pm SEM. Paired $t$ tests were performed on raw data. ${ }^{*} p<0.05$ ( $t$ test).

A

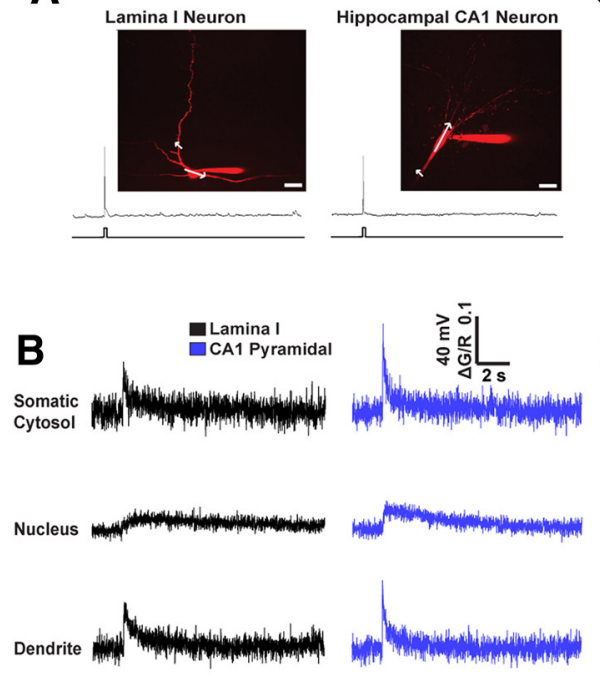

C

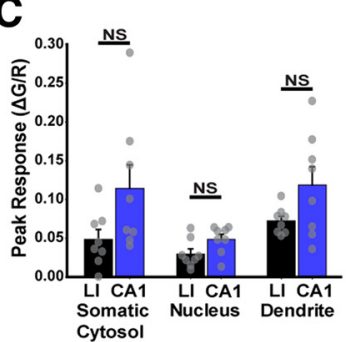

E

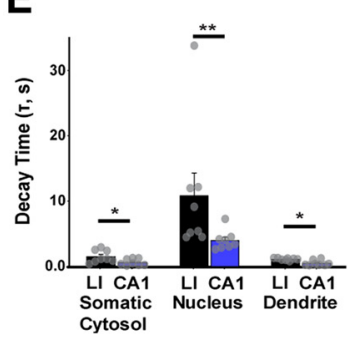

D

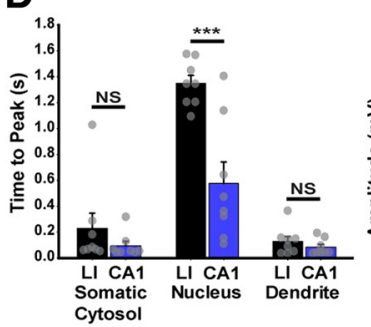

$F$

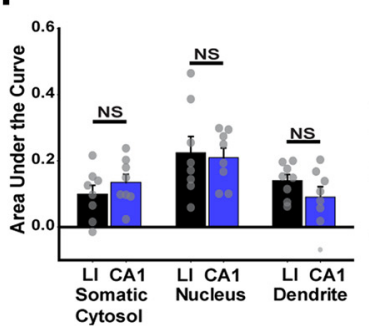

G

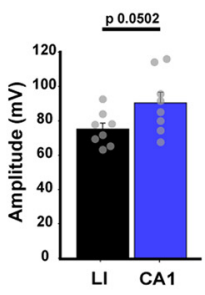

H

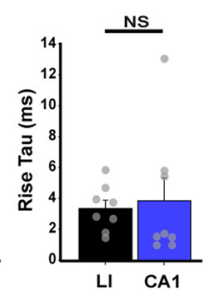

I

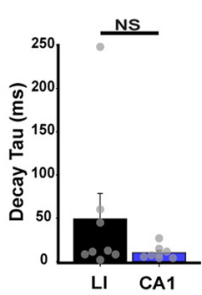

J

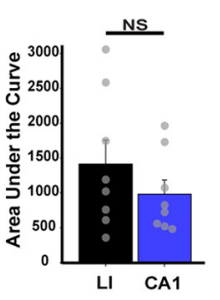

Figure 8. Lamina I calcium responses exhibit slower rise and decay kinetics compared with CA1 pyramidal neurons. $\boldsymbol{A}$, Example two-photon images of a spinal cord lamina I neuron (left) and hippocampal CA1 pyramidal neuron (right). White arrows indicate line scan selections. Bottom, Current-clamp traces and current injection traces for these example neurons. Scale bars, $20 \mu$ m. $\boldsymbol{B}$, Mean calcium responses for somatic cytosol, nucleus, and dendritic calcium responses evoked by single AP firing for lamina I neurons (black, LI, $n=8$ neurons) and hippocampal CA1 neurons (blue, CA1, $n=8$ neurons). Calibration: $y$ axis $=40 \mathrm{mV}, \Delta G / R 0.1, x$ axis $=2 \mathrm{~s}$. C, Mean peak response of $\Delta G / R$ responses for all three subcellular compartments. $t$ tests were performed for each compartment: cytosol $p=0.065$, nucleus $p=0.055$, dendrite $p=0.279$. $\boldsymbol{D}$, Mean time to peak. $t$ tests were performed for each compartment: cytosol $p=0.064$, nucleus $p<0.001$, dendrite $p=0.645$. $\boldsymbol{E}$, Mean decay time constant $(\tau)$. $t$ tests were performed for each compartment: cytosol $p=0.035$, nucleus $p=0.005$, dendrite $p=0.01$. $\boldsymbol{F}$, Mean AUC. $t$ tests were performed for each compartment: cytosol $p=0.349$, nucleus $p=0.793$, dendrite $p=0.192$. $\mathbf{C}-\boldsymbol{F}, n=8$ neurons for each cell type. Data are mean \pm SEM. ${ }^{*} p<0.05 ;{ }^{* *} p<0.01$; ${ }^{* * *} p<0.005 ; t$ tests. G-J, Comparison of AP characteristics between lamina I and CA1 hippocampal pyramidal neurons. Quantification of $(\boldsymbol{G})$ peak AP amplitude $(p=0.05),(\boldsymbol{H})$ rise $\tau(p=0.505),(\boldsymbol{I})$ decay $\tau(p=0.279)$, and $(\boldsymbol{J})$ AUC $(p=0.296)$ between the two cell types ( $N=8$ neurons for each cell type). Data are mean \pm SEM. Unpaired $t$ tests were performed for each comparison.

compartment of lamina I neurons, which is characterized by exceptionally long rise and decay components.

Calcium responses in dendrites are mediated by active backpropagation in lamina I neurons

The AP-evoked $\Delta G / R$ responses we observed in the dendrites could potentially be mediated by passive diffusion of calcium from the soma as a consequence of somatically generated APs, or by active processes in the dendrites. To distinguish between these two possibilities, we measured $\Delta G / R$ responses to single APs at several distances along dendrites in lamina I neurons with sufficiently long dendritic arbor (Fig. 9). We found no significant changes in the amplitude of the peak (Kruskal-Wallis one-way ANOVA, $p=0.916, n=9, n=6, n=8$, and $n=6$ neurons at 20, 

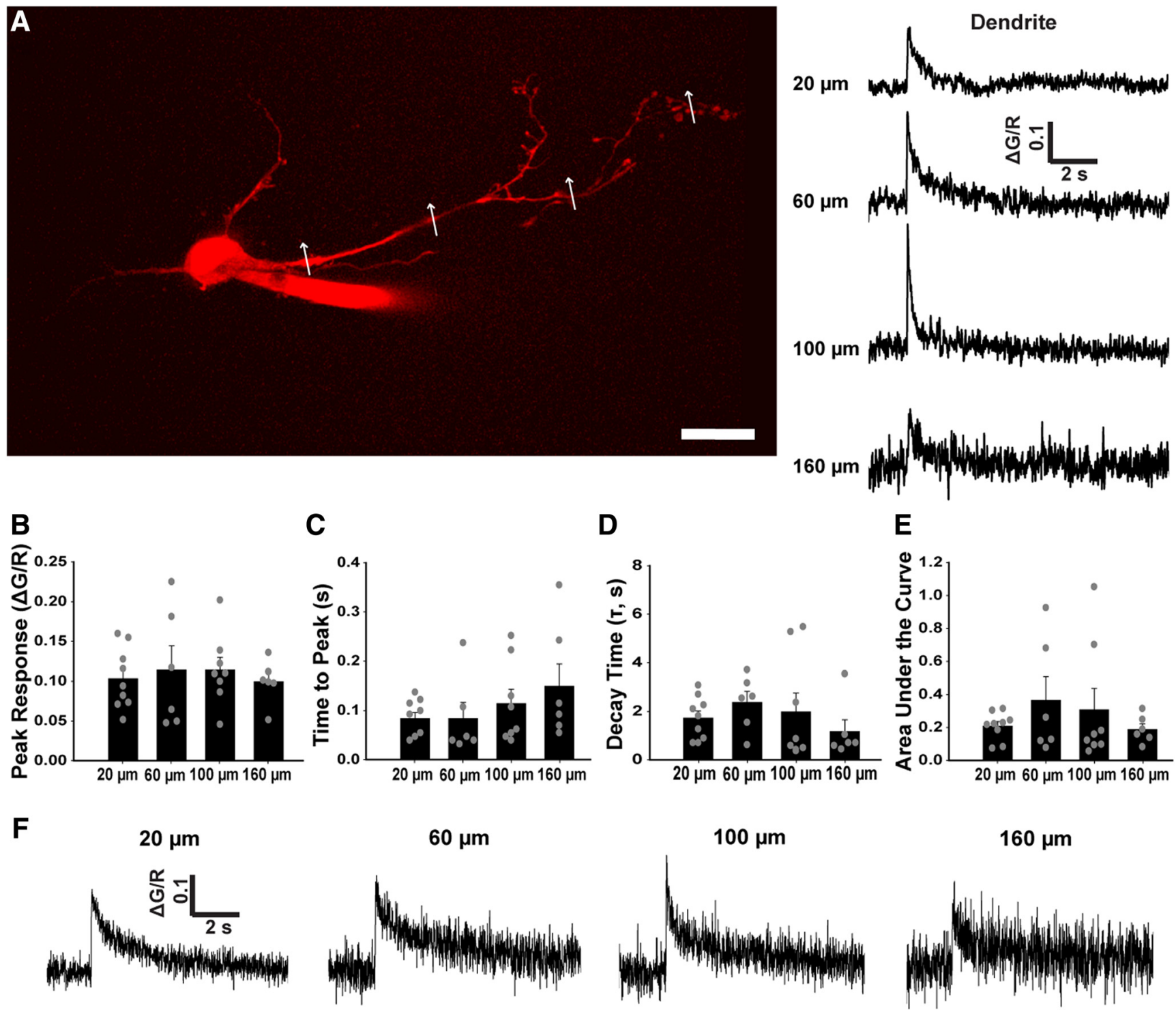

G

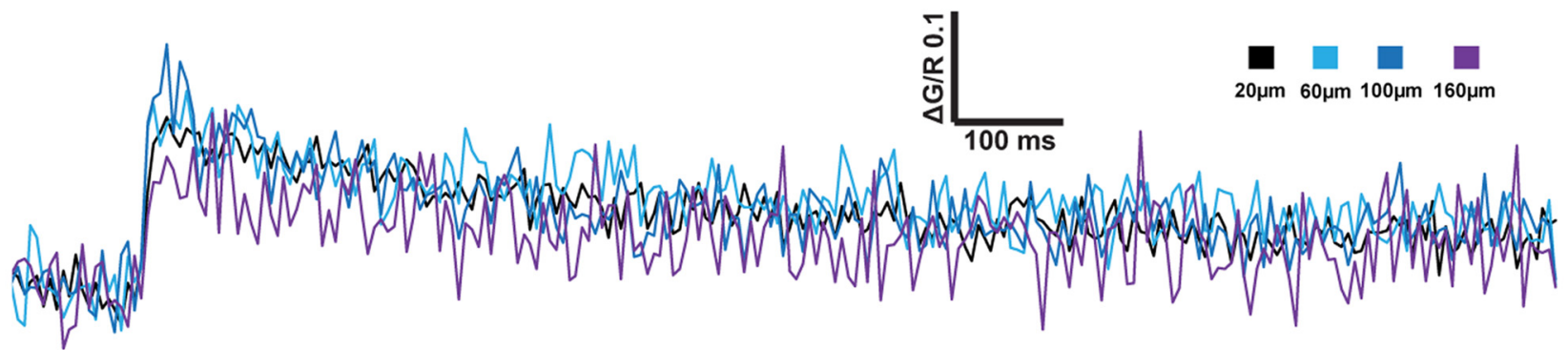

Figure 9. Calcium responses in dendrites are mediated by active backpropagation in lamina I neurons. $\boldsymbol{A}$, Left, Two-photon image of a lamina I neuron, with line scan selections (white arrows). Scale bar, $20 \mu \mathrm{m}$. Right, Mean calcium responses to single APs in dendritic segments of this example neuron (mean of 2-5 line scans per Rol). Calibration: $y$ axis $=\Delta \mathrm{G} / \mathrm{R} 0.1, x$ axis $=2$ s. $\boldsymbol{B}$, Mean peak responses in dendrites at each distance away from the soma. One-way ANOVA, $p=0.916$. C, Mean time to peak in dendrites at each distance away from the soma. One-way ANOVA, $p=0.349$. $\boldsymbol{D}$, Mean decay time constant $(\tau)$ in dendrites at each distance away from the soma. Kruskal-Wallis one-way ANOVA, $p=0.281$. $\boldsymbol{E}$, Mean AUC in dendrites at each distance away from the soma. Kruskal-Wallis one-way ANOVA, $p=0.560$. $\boldsymbol{B}-\boldsymbol{E}$, No post hoc $t$ tests were performed due to lack of significant effect of distance. $\boldsymbol{F}$, Mean calcium responses in dendrites at 20,60, 100, and $160 \mu \mathrm{m}$ away from the soma. Calibration: $y$ axis $=\Delta \mathrm{G} / \mathrm{R} 0.07, x$ axis $=2 \mathrm{~s}$. G, Left, Overlay of mean calcium responses in $\boldsymbol{F}$. Calibration: $y$ axis $=\Delta \mathrm{G} / \mathrm{R} 0.1, x$ axis $=100 \mathrm{~ms}$. $\boldsymbol{B}-\boldsymbol{G}, n=9, n=6, n=8$, and $n=7$ dendrites from 8,5,7, and 6 neurons for 20,60, 100, and $160 \mu \mathrm{m}$ from the soma, respectively. Error bars indicate SEM.

60,100 , and $160 \mu \mathrm{m}$, respectively) nor of the time to peak (Kruskal-Wallis one-way ANOVA, $p=0.349$ ) of the $\Delta \mathrm{G} / \mathrm{R}$ responses at distances of $20,60,100$, and $160 \mu \mathrm{m}$ away from the soma. Moreover, there were no distance-dependent differences in the decay times (Kruskal-Wallis one-way ANOVA, $p=0.281$ ) or in AUC (Kruskal-Wallis one-way ANOVA, $p=0.560$ ).

In the case of passive diffusion of $\mathrm{Ca}^{2+}$, the amplitude of the $\Delta G / R$ response is predicted to decrease greatly with distance 
from the soma and the time to peak is predicted to be prolonged. With active backpropagation, the amplitude of the $\Delta \mathrm{G} / \mathrm{R}$ response will be governed by the relative expression of voltagegated ion channels in each branch of the dendritic arbor (Spruston et al., 1995; Williams and Stuart, 2000; Waters et al., 2005), and could therefore avoid the amplitude degradation seen with passive diffusion. Additionally, with active backpropagation, the time to peak would be predicted to remain constant within the time resolution of our experiments (Markram et al., 1995; Williams and Stuart, 2000; Golding et al., 2001; Casale and McCormick, 2011). Thus, the consistency in amplitude and time to peak of $\Delta \mathrm{G} / \mathrm{R}$ responses along lamina I dendrites eliminates the possibility that the responses are mediated by passive diffusion of calcium from the soma, and instead imply that dendritic responses are mediated by active AP backpropagation.

\section{Calcium responses differentiate bursts from single APs in lamina I neurons}

In a number of brain regions, including cortex, hippocampus, and cerebellum (Liao and Lien, 2009; Tada et al., 2014), calcium responses evoked by bursts of APs have been reported to encode their number and frequency. We therefore wondered whether calcium responses in spinal cord lamina I neurons can detect and encode AP bursts. To this end, we evoked single APs or bursts containing 2 or $4 \mathrm{APs}$, at an interval of $30 \mathrm{~s}$ between stimuli $(0.033 \mathrm{~Hz})$. For stimuli comprised of 2 or $4 \mathrm{APs}$, the intraburst frequency was $0.5,10$, or $50 \mathrm{~Hz}$. We measured the maximum amplitude of the $\Delta \mathrm{G} / \mathrm{R}$ response and the AUC for each condition (Fig. 10). In three subcellular compartments (somatic cytosol, nucleus, and dendrites), we examined the relationship between maximum $\Delta \mathrm{G} / \mathrm{R}$ or AUC with frequency of firing and number of APs.

Overall, across the frequency range in each of the compartments, the maximum $\Delta \mathrm{G} / \mathrm{R}$ was greater with bursts of 2 or 4 APs compared with singlets in all three compartments. With bursts of APs, there was an increase in maximum $\Delta \mathrm{G} / \mathrm{R}$ with increasing number of APs. Any frequency-dependent increase in maximum $\Delta \mathrm{G} / \mathrm{R}$ largely plateaued at $0.5 \mathrm{~Hz}$ in all three compartments, with a significant frequency-dependent increase in maximum $\Delta G / R$ only within the somatic cytosol (two-way ANOVAs, $p=0.042$ for somatic cytosol, $p=0.014$ for nucleus, $p=0.005$ for dendrite with number of APs as a factor; $p=0.034$ for somatic cytosol, $p=0.386$ for nucleus, $p=0.133$ for dendrite with frequency as a factor).

As with the maximum $\Delta \mathrm{G} / \mathrm{R}$, the AUC was greater with bursts of 2 or 4 APs compared with singlets in all three compartments, but no significant differences were found in AUC with increasing firing frequency (two-way ANOVAs, $p<0.001$ for somatic cytosol, $p=0.039$ for nucleus, $p=0.008$ for dendrite with number of APs as a factor; $p=0.906$ for somatic cytosol, $p=0.801$ for nucleus, $p=0.696$ for dendrite with frequency as a factor).

Together, these data indicate that both maximum $\Delta \mathrm{G} / \mathrm{R}$ and AUC distinguish single APs at low frequency $(0.033 \mathrm{~Hz})$ from bursts containing $\geq 2$ APs. From this, we infer that calcium responses in the somatic cytosol, the nucleus, and the dendrites of lamina I neurons detect multi-AP bursts. Moreover, while the maximum $\Delta \mathrm{G} / \mathrm{R}$ and AUC increased concomitantly from 1-2 to 4 APs, we observed only minor changes with increasing frequency. Therefore, the major determinant for both maximum $\Delta \mathrm{G} / \mathrm{R}$ and AUC is the number of APs, with the calcium response effectively counting APs within this range.

\section{Single AP-evoked calcium responses invade dendritic spines of lamina I neurons}

Spines on dendrites are sites of excitatory synaptic inputs and form a biochemically and spatially distinct subcellular compartment from dendrites (Nimchinsky et al., 2002; Bloodgood and Sabatini, 2007). Dendritic spines are present on many neurons in lamina I of the dorsal horn (Lima and Coimbra, 1986; Galhardo and Lima, 1999; Cordero-Erausquin et al., 2009), although the spine density is lower compared with, for example, CA1 pyramidal neurons. An outstanding question is whether lamina I dendritic spine $\mathrm{Ca}^{2+}$ concentration can be affected by neuronal activity.

To address this question, we compared dendritic spine $\Delta \mathrm{G} / \mathrm{R}$ responses with those from its parent dendrite (Fig. 11). In every dendritic spine recorded ( $n=22$ spines in 22 neurons), we found that evoking a single AP caused a reproducible, time-locked increase in $\Delta \mathrm{G} / \mathrm{R}$. The peak and AUC of dendritic spine $\Delta \mathrm{G} / \mathrm{R}$ responses were smaller than adjacent dendritic $\Delta \mathrm{G} / \mathrm{R}$ responses (paired $t$ tests, $p<0.001$ and $p=0.005$, respectively, $n=22$ neurons). However, we observed no delay in onset of the $\Delta G / R$ response (Fig. 11C), as well as no significant difference in the time to peak and decay time of the $\Delta \mathrm{G} / \mathrm{R}$ responses between dendritic spines and their parent dendrites (paired $t$ tests, $p=0.770$ and $p=0.825$, respectively). Thus, we conclude that an AP initiated at the soma evokes a tightly time-locked increase in $\mathrm{Ca}^{2+}$ concentration in the dendritic spines, as well as in the dendrites of lamina I neurons.

\section{AP-evoked calcium responses are similar across subpopulations of lamina I neurons}

Lamina I neurons are both electrophysiologically and morphologically heterogeneous, with subpopulations defined by AP firing pattern in response to intracellular current injection, or by number of primary dendrites (Lima and Coimbra, 1986; Prescott and De Koninck, 2002; Ruscheweyh and Sandkuhler, 2002; Polgar et al., 2013; Punnakkal et al., 2014). We investigated whether the $\Delta \mathrm{G} / \mathrm{R}$ responses to single APs might differ in the various subpopulations of lamina I neurons. Based on firing pattern in response to current injection, four types of lamina I neurons have been identified (Prescott and De Koninck, 2002; Punnakkal et al., 2014): delayed onset, single spike, phasic, and tonic. In the population of 210 neurons studied, we found that $53 \%$ were tonic firing, $22 \%$ delayed onset, $14 \%$ phasic, and $4 \%$ single spike firing (Fig. 12A). The remaining $7 \%$ of neurons could not be classified.

The average peak response, rise time, decay time, and AUC in each compartment (cytosol, nucleus, dendrite, and dendritic spine) were calculated and compared between each firing type (Fig. 12E-H). Across these many comparisons, we found only one statistically significant difference: where single spike neurons possess a faster nuclear rise time $(0.80 \pm 0.13 \mathrm{~s})$ than other neuron types (delayed firing $=1.23 \pm 0.06 \mathrm{~s}$, phasic firing $=1.12 \pm 0.07 \mathrm{~s}$, tonic firing $=1.23 \pm 0.05 \mathrm{~s}$ ) (Kruskal-Wallis one-way ANOVA, $p=0.018, n=46$ neurons for delayed, $n=29$ neurons for phasic, $n=8$ neurons for single spike, and $n=112$ neurons for tonic firing). Therefore, grouping lamina I neurons by firing type revealed that the $\Delta G / R$ responses were largely similar in these different subpopulations.

To investigate possible differences in $\Delta \mathrm{G} / \mathrm{R}$ responses in morphologically defined neuronal subpopulations, we assigned neurons to subpopulations based on previous classification schemes (Lima and Coimbra, 1986) (Fig. 12B). In the population studied, $34 \%$ of neurons were fusiform, $33 \%$ multipolar, $25 \%$ pyramidal, $0 \%$ flattened, and $8 \%$ unclassified. We found no significant differences in $\Delta \mathrm{G} / \mathrm{R}$ responses across these morphologically defined 

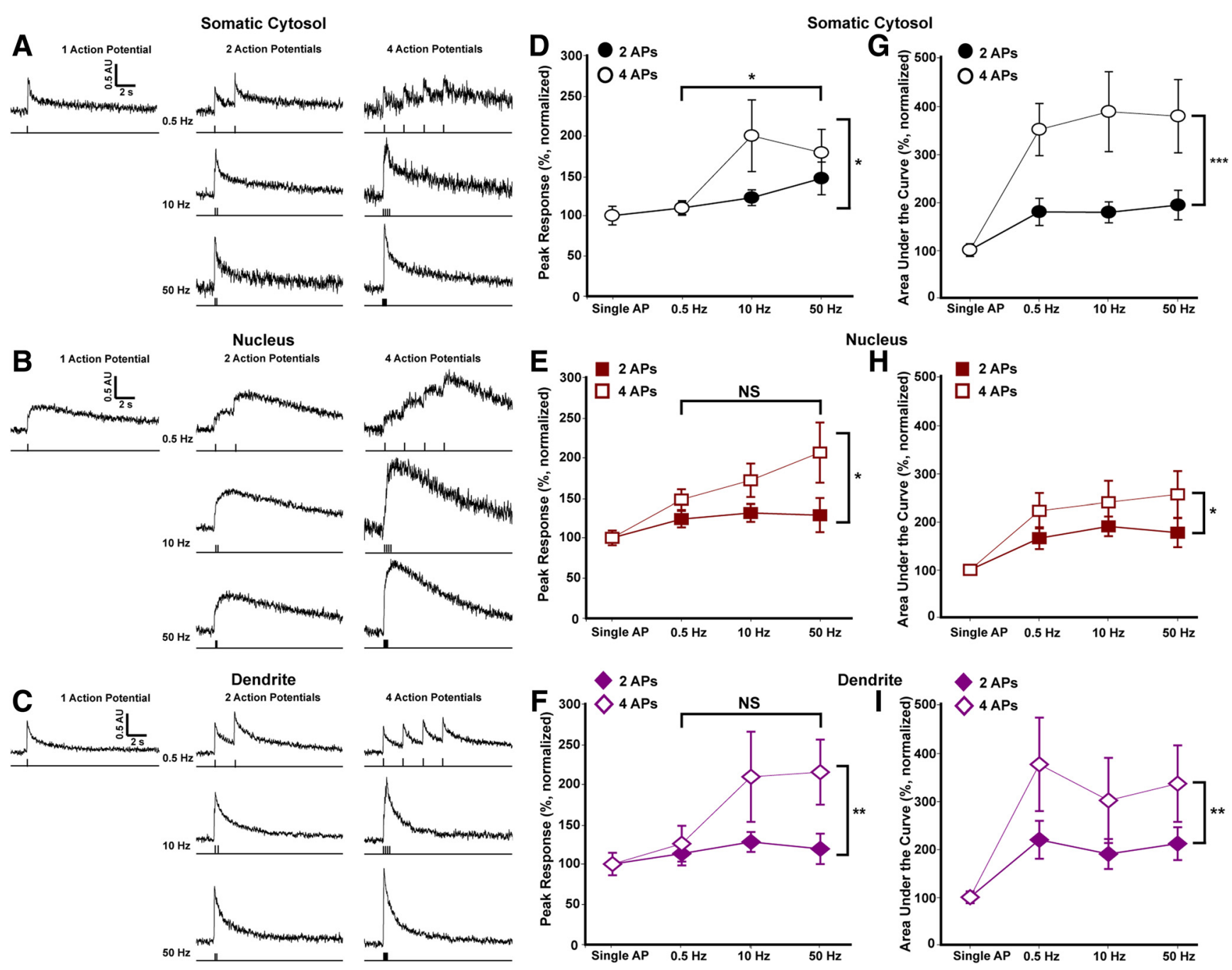

Figure 10. Calcium responses encode number of APs and firing frequency. One, 2, or 4 APs were evoked through $5 \mathrm{~ms}$ current injection in lamina I neurons at $0.5,10$, or $50 \mathrm{~Hz}$. A, Mean somatic cytosol calcium responses, normalized to the calcium response to a single AP for each neuron $(n=20, n=13, n=17, n=10, n=4, n=5$, and $n=9$ neurons for single AP, 2 AP $0.5 \mathrm{~Hz}$, $2 \mathrm{AP} 10 \mathrm{~Hz}, 2 \mathrm{AP} 50 \mathrm{~Hz}, 4 \mathrm{AP} 0.5 \mathrm{~Hz}, 4 \mathrm{AP} 10 \mathrm{~Hz}$, and $4 \mathrm{AP} 50 \mathrm{~Hz}$, respectively). Calibration: $y$ axis $=0.5 \mathrm{AU}, x$ axis $=2 \mathrm{~s}$. B, Data presented as in $\boldsymbol{A}$, but for nuclear calcium responses ( $n=20$, $n=13, n=18, n=10, n=4, n=5$, and $n=9$ neurons for single AP, $2 \mathrm{AP} 0.5 \mathrm{~Hz}, 2 \mathrm{AP} 10 \mathrm{~Hz}, 2 \mathrm{AP} 50 \mathrm{~Hz}, 4 \mathrm{AP} 0.5 \mathrm{~Hz}, 4 \mathrm{AP} 10 \mathrm{~Hz}$, and $4 \mathrm{AP} 50 \mathrm{~Hz}$, respectively). Calibration: $y$ axis $=0.4 \mathrm{AU}$, $x$ axis $=2$ s. C, Data presented as in $A$, but for dendritic calcium responses $(n=20, n=14, n=18, n=11, n=4, n=4$, and $n=8$ neurons for single AP, $2 \mathrm{AP} 0.5 \mathrm{~Hz}, 2 \mathrm{AP} 10 \mathrm{~Hz}, 2 \mathrm{AP} 50 \mathrm{~Hz}$, $4 \mathrm{AP} 0.5 \mathrm{~Hz}, 4 \mathrm{AP} 10 \mathrm{~Hz}$, and $4 \mathrm{AP} 50 \mathrm{~Hz}$, respectively). Calibration: $y$ axis $=0.5 \mathrm{AU}, x$ axis $=2 \mathrm{~s}$. D, Quantification of peak calcium responses in the somatic cytosol. A two-way ANOVA on ranks was performed with number of APs and firing frequency as factors (interaction: $F_{(2,0.56)}=1.537, p=0.225$; number of APs: $F_{(1,1.58)}=4.34, p=0.042$; firing frequency: $F_{(2,1.31)}=3.60$, $p=0.034)$, determining that both number of APs and firing frequency were significant factors. $\boldsymbol{E}$, Quantification of peak calcium responses in the nucleus. A two-way ANOVA on ranks was performed with number of APs and firing frequency as factors (interaction: $F_{(2,0.31)}=775, p=0.466$; number of APs: $F_{(1,2.63)}=6.46, p=0.014$; firing frequency: $\left.F_{(2,0.395)}=0.39, p=0.386\right)$, determining that number of APs was a significant factor, but not firing frequency. $F$, Quantification of peak calcium responses in the dendrite. A two-way ANOVA on ranks was performed with number of APs and firing frequency as factors (interaction: $F_{(2,0.69)}=1.39, p=0.259$; number of APs: $F_{(1,4.26)}=8.59, p=0.005$; firing frequency: $\left.F_{(2,1.04)}=2.09, p=0.133\right)$, determining that number of APs was a significant factor, but not firing frequency. $G$, Quantification of AUC of calcium responses in the somatic cytosol. A two-way ANOVA on ranks was performed with number of APs and firing frequency as factors (interaction: $F_{(2,0.13)}=0.07, p=0.929$; number of APs: $F_{(1,40)}=22.359, p<0.001$; firing frequency: $\left.F_{(2,0.18)}=0.10, p=0.906\right)$, determining that number of APs was a significant factor, but not firing frequency. $\boldsymbol{H}$, Quantification of AUC of calcium responses in the nucleus. A two-way ANOVA on ranks was performed with number of APs and firing frequency as factors (interaction: $F_{(2,0.1)}=0.11, p=0.899$; number of APs: $F_{(1,4)}=4.49, p=0.039$; firing frequency: $F_{(2,0.2)}=0.22, p=0.801$ ), determining that number of APs was a significant factor, but not firing frequency. $I$, Quantification of AUC of calcium responses in the dendrite. A two-way ANOVA on ranks was performed with number of APs and firing frequency as factors (interaction: $F_{(2,0.17)}=0.07, p=0.930$; number of APs: $F_{(1,18)}=7.64, p=0.008$; firing frequency: $\left.F_{(2,0.87)}=0.36, p=0.696\right)$, determining that number of APs was a significant factor, but not firing frequency. $\boldsymbol{A}-\boldsymbol{C}$, Data are normalized mean. $\boldsymbol{D}-\boldsymbol{I}$, Data are normalized mean $\pm \mathrm{SEM} .{ }^{*} p<0.05 ;{ }^{* *} p<0.01 ;{ }^{* * *} p<0.005 ; t$ tests.

subpopulations (Fig. 12I-L). Together, these findings indicate that AP-evoked calcium responses are nearly identical across the different electrophysiologically and morphologically defined subpopulations of lamina I neurons.

\section{Discussion}

AP-evoked calcium responses in lamina I neurons

Here we have described calcium responses that invade the somatic cytosol, nucleus, dendrites, and dendritic spines in $>99 \%$ of lamina I neurons in response to single APs. These AP-evoked calcium responses were mediated primarily by influx of calcium through VGCCs. In the somatic cytosol and the nucleus, calcium influx was amplified by CICR from ryanodine-sensitive calcium stores.

The kinetics of the AP-evoked calcium responses in the somatic cytosol, dendrites, and dendritic spines were similar to one another; relative calcium concentration peaked $150-200 \mathrm{~ms}$ after AP firing and decayed with a time constant of $\sim 1.5 \mathrm{~s}$. By contrast, the kinetics of the nuclear calcium responses differed 


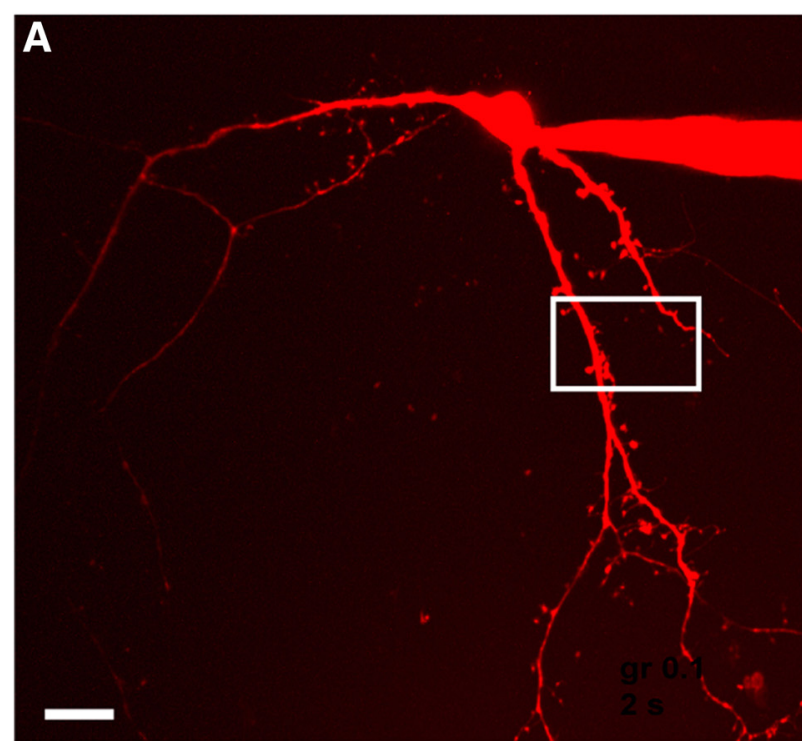

C Average Dendritic Response

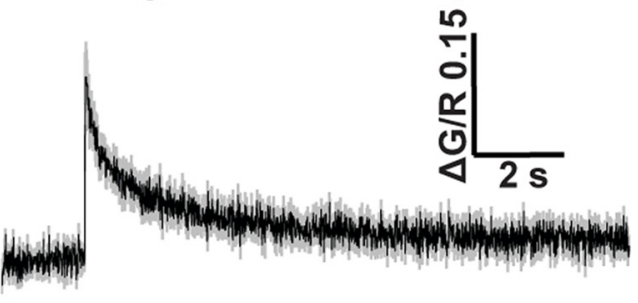

Average Dendritic Spine Response
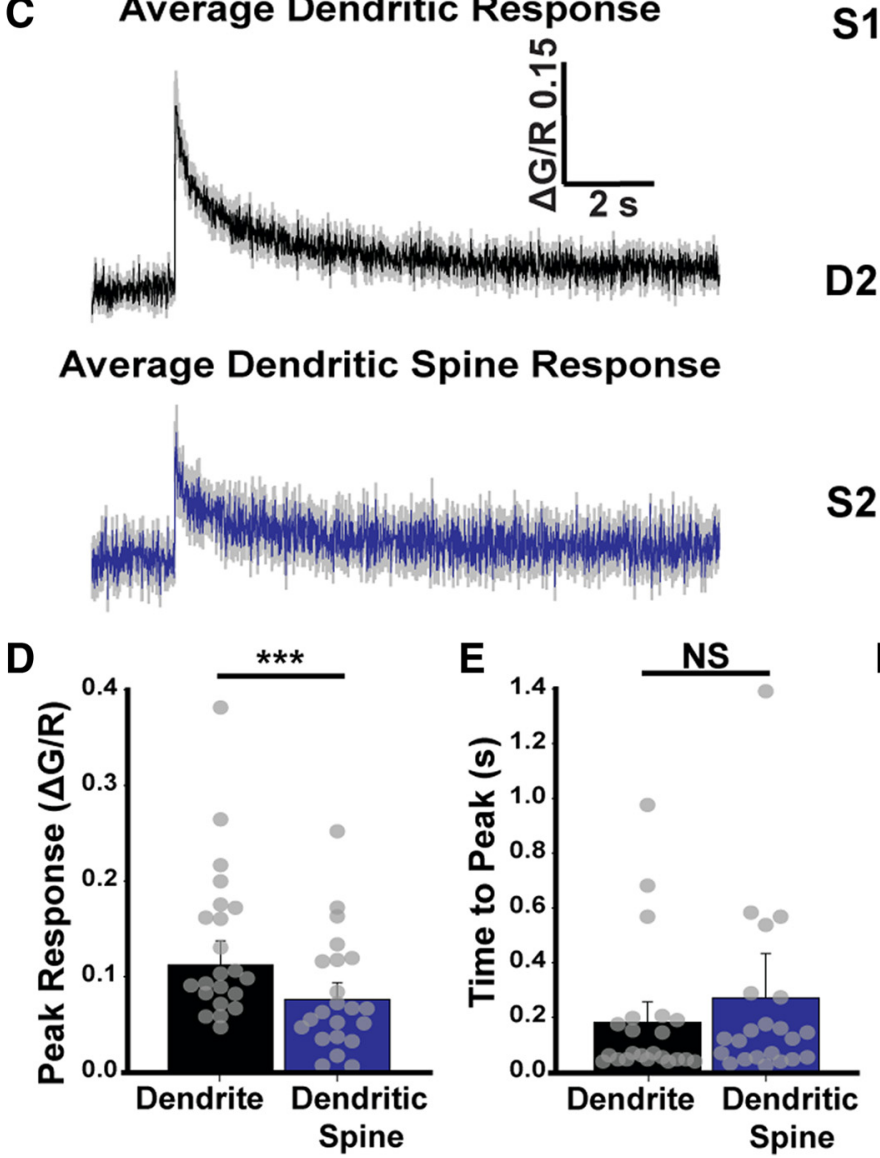

D2

S2
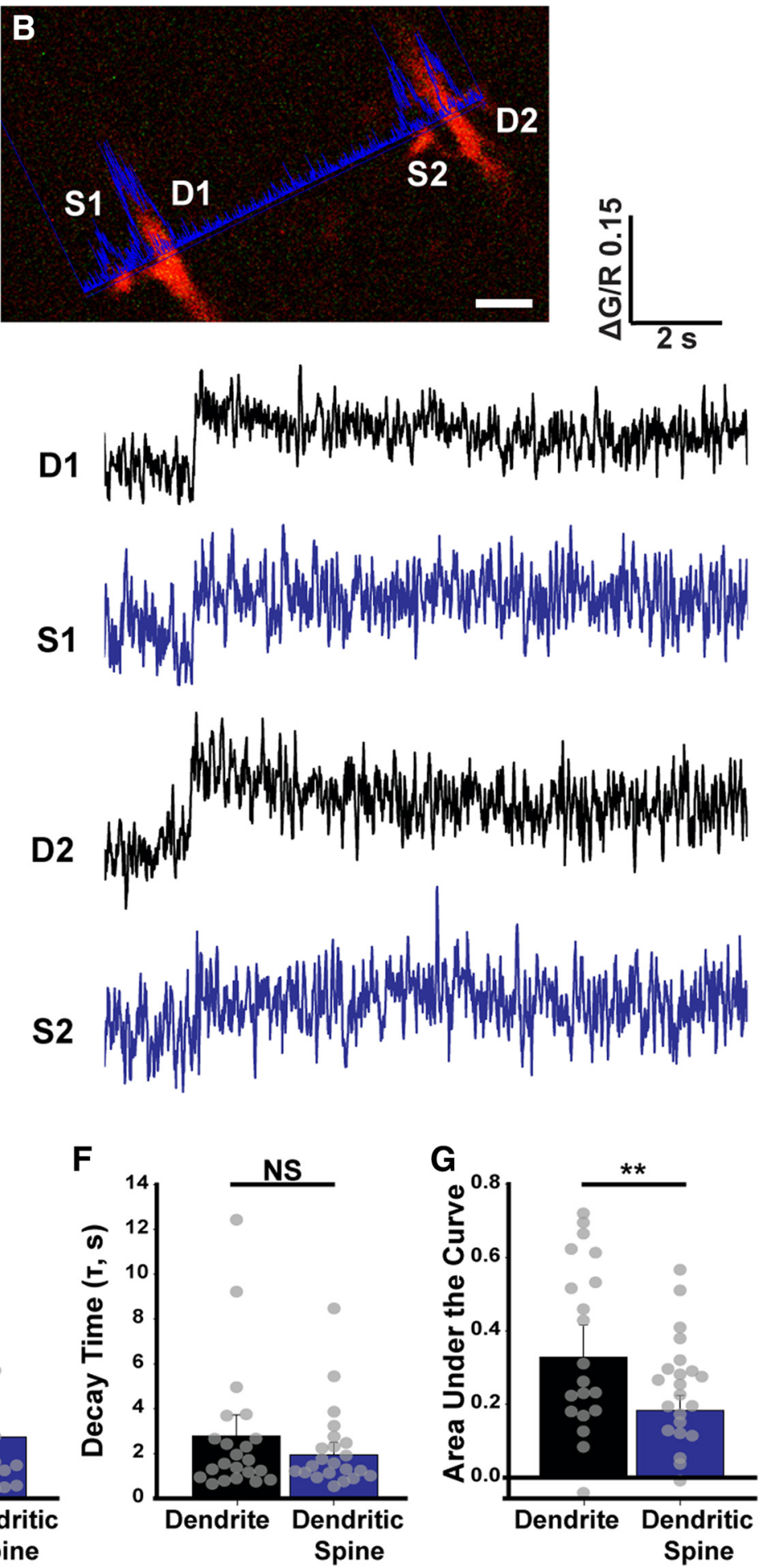

Figure 11. Single AP-evoked calcium responses invade dendritic spines of lamina I neurons. $\boldsymbol{A}$, Flattened z-stack image of a lamina I neuron. White box represents location of inset. Scale bar, $20 \mu \mathrm{m}$. B, Top, Frame scan image delineating line scan selection of two primary dendrites and adjacent dendritic spines. Scale bar, $3 \mu \mathrm{m}$. Bottom, Single AP-evoked calcium responses in each compartment of the depicted line scan. Responses displayed as mean of 2-7 line scans, smoothed by a $5 \times$ running average. D1, D2, Dendritic compartments; S1, S2, dendritic spine compartments. Calibration: $y$ axis $=\Delta G / R$ 0.15, $x$ axis $=2 \mathrm{~s}$. C, Mean $\Delta \mathrm{G} / \mathrm{R}$ responses in dendrites and dendritic spines $(N=22$ neurons $) \pm S E M$. Calibration: $y$ axis $=\Delta G / R$ 0.1, $x$ axis $=2 \mathrm{~s}$. $D-$ $\boldsymbol{G}$, Quantification of dendritic and dendritic spine calcium responses. $\boldsymbol{D}$, Peak calcium response. Paired $t$ test, $p<0.001$. $\boldsymbol{E}$, Time to peak. Paired $t$ test, $p=0.770$. $\boldsymbol{F}$, Decay time constant $(\tau)$. Paired $t$ test, $p=0.627$. G, AUC. Paired $t$ test, $p=0.005$. D-G, Data are mean \pm SEM. Paired $t$ tests were performed for each measure. ${ }^{* *} p<0.01 .{ }^{* * *} p<0.005$.

substantially, having an initial fast rise and secondary slow rise component, and not reaching peak until $>1 \mathrm{~s}$ after the AP. The nuclear decay time was also substantially longer than any other compartment, with a time constant $>10$ s. In all compartments, these rise and decay times were markedly longer than previously reported values in cortical and hippocampal neurons (Sandler and Barbara, 1999; Golding et al., 2001; Power and Sah, 2002; Rozsa et al., 2004; Tonini et al., 2013). The differences we 
A Delayed Onset

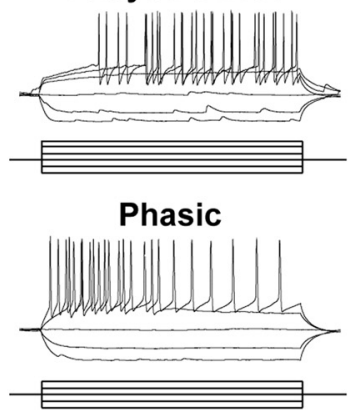

Single Spike
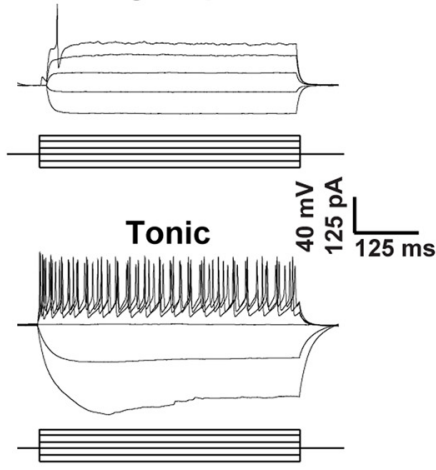

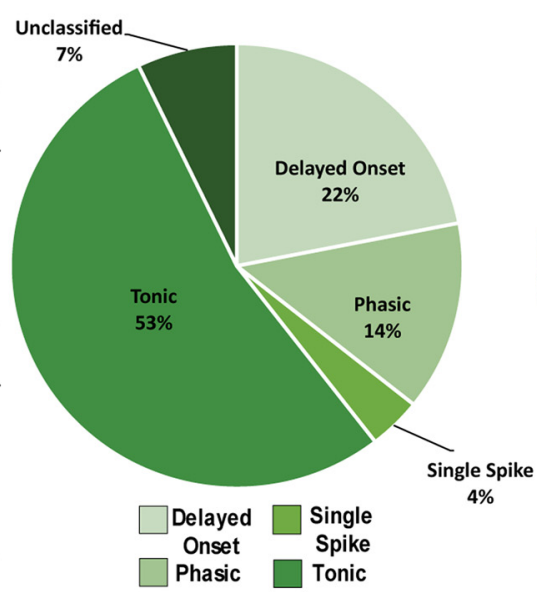

C Single AP Current Injection Threshold by Firing Type
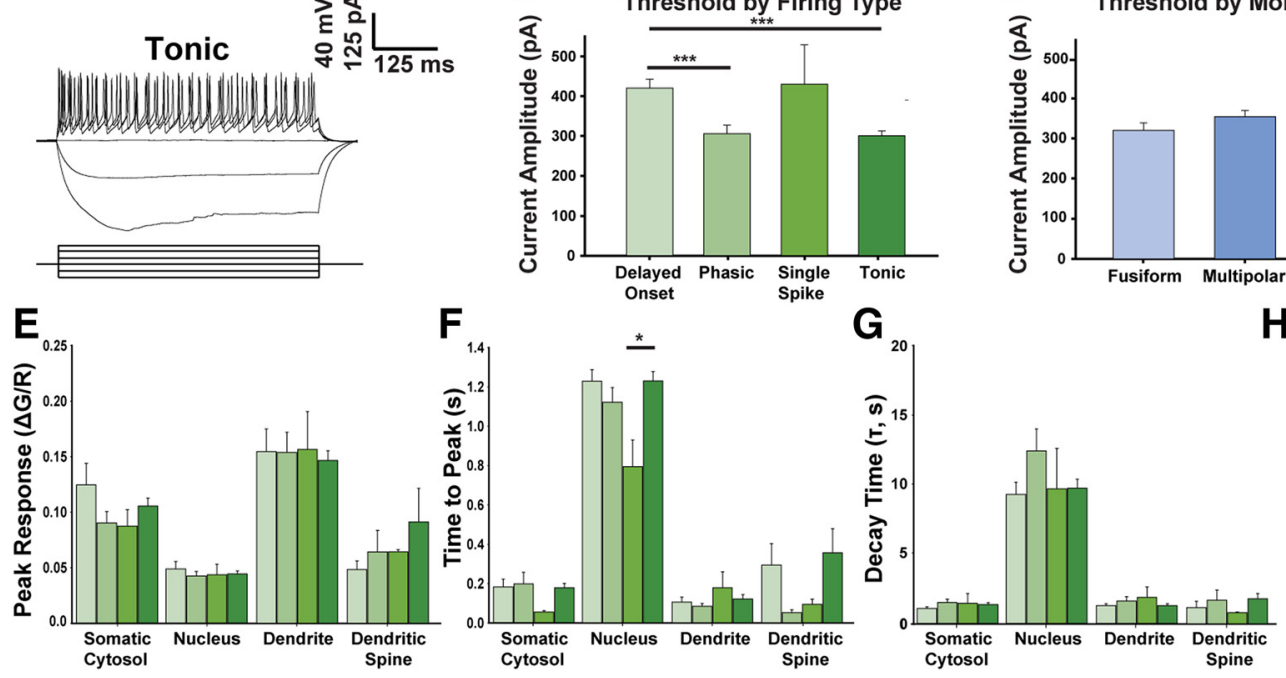

D Single AP Current Injection

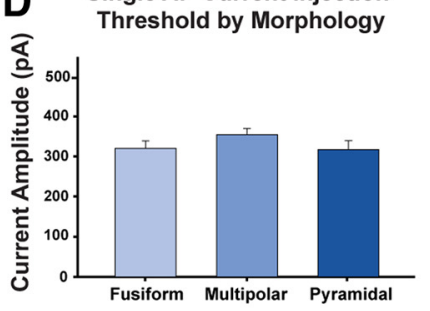

H

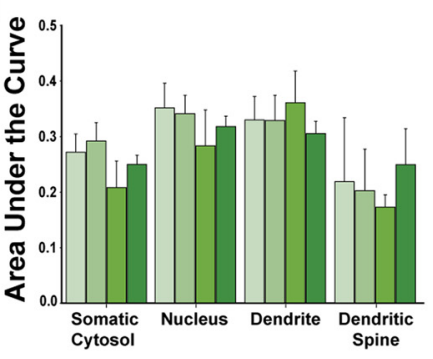

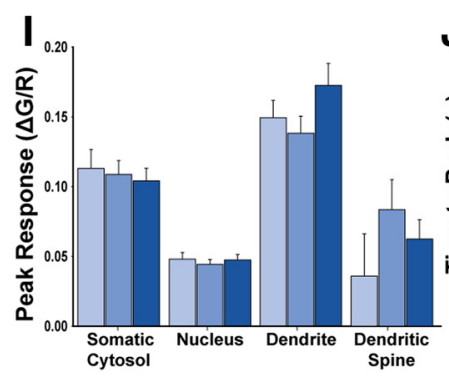
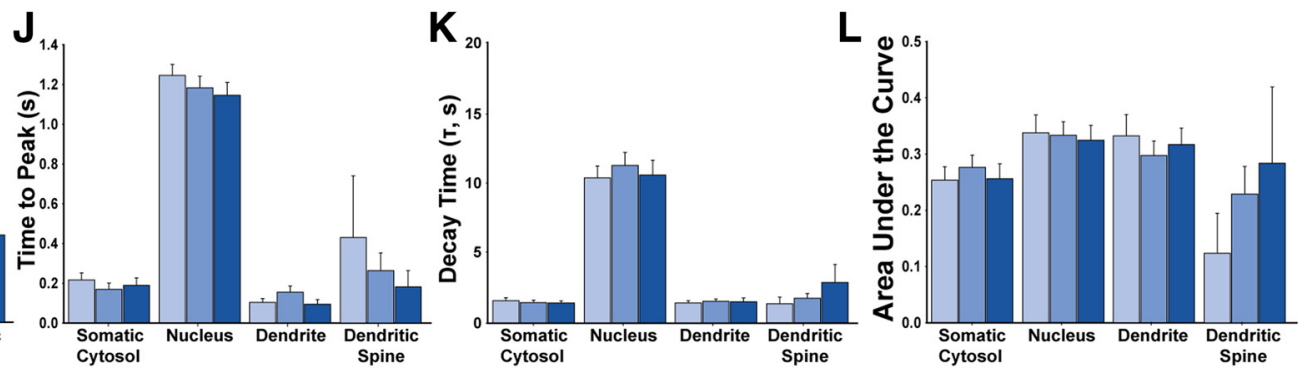

Figure 12. AP-evoked calcium responses are similar across subpopulations of lamina I neurons. $\boldsymbol{A}$, Example voltage-current curves displaying the four major firing type classifications, and the population distribution of these firing types ( $n=210$ neurons total; delayed onset, phasic, single spike, tonic, and unclassified: $n=46, n=29, n=8, n=112$, and $n=15$ neurons, respectively). Calibration: $y$ axis $=40 \mathrm{mV}, 125 \mathrm{pA} ; x$ axis $=125 \mathrm{~ms}$. $\boldsymbol{B}$, Example two-photon images displaying the three major morphology classifications, and the population distribution of these morphologies ( $n=210$ neurons total; fusiform, multipolar, pyramidal, and unclassified: $n=71, n=70, n=53$, and $n=16$ neurons, respectively). Scale bars, $20 \mu \mathrm{m}$. C, Separating neurons by firing type yielded significant differences in a Kruskal-Wallis one-way ANOVA $(p<0.001)$ comparing mean current injection amplitude $(5 \mathrm{~ms}$ duration) required to yield an AP at $100 \%$ fidelity (delayed onset, phasic, single spike, and tonic: $n=46, n=27, n=7$, and $n=93$ neurons, respectively). Post hoc Tukey $t$ tests revealed significant differences in the delayed onset firing type, which requires a larger current amplitude than phasic or tonic firing neurons to yield an $\operatorname{AP}(p<0.001$ and $p<0.001$, respectively). $D$, Separating neurons by morphology yielded significant differences in a Kruskal-Wallis one-way ANOVA ( $p=0.028$ ) comparing mean current injection amplitude (fusiform, multipolar, and pyramidal: $n=65, n=65$, and $n=44$ neurons, respectively). However, post hoc Tukey $t$ tests revealed no significant differences between morphologies ( $p=1.00$ for pyramidal vs fusiform, $p=0.079$ for pyramidal vs multipolar, $p=0.055$ for fusiform vs multipolar). $\boldsymbol{E}-\boldsymbol{H}$, Quantification of calcium responses in all four subcellular compartments split by firing type. For somatic cytosol, nucleus, and dendrite: $n=195$ neurons total; delayed onset, phasic, single spike, and tonic: $n=46, n=29, n=8$, and $n=112$ neurons, respectively. For dendritic spine: $n=16$ neurons total; delayed onset, phasic, single spike, and tonic: $n=4, n=2, n=2$, and $n=8$ neurons, respectively. $\boldsymbol{E}$, Mean peak response. Kruskal-Wallis one-way ANOVAs for somatic cytosol $p=0.719$, for nucleus $p=0.796$, for dendrite $p=0.702$, for dendritic spine $p=0.951$. $\boldsymbol{F}$, Time to peak. Kruskal-Wallis one-way ANOVAs for somatic cytosol $p=0.271$, for nucleus $p=0.018$, for dendrite $p=0.813$, for dendritic spine $p=0.431$. Post hoc $t$ test, $p=0.027$ for comparison between single spike and tonic firing neurons, post-Bonferroni correction. $\mathbf{G}$, Decay time constant $(\tau)$. Kruskal-Wallis one-way ANOVAs for somatic cytosol $p=0.265$, for nucleus $p=0.449$, for dendrite $p=0.723$, for dendritic spine $p=0.510$. $\boldsymbol{H}$, AUC. Kruskal-Wallis one-way ANOVAs for somatic cytosol $p=0.719$, for nucleus $p=0.796$, for dendrite $p=0.702$, for dendritic spine $p=0.951$. $I-\mathbf{L}$, Quantification of calcium responses in all four subcellular compartments split by morphology. For somatic cytosol, nucleus, and dendrite: $n=195$ neurons total; fusiform, multipolar, and pyramidal: $n=71, n=70$, and $n=53$ neurons, respectively. For dendritic spine: $n=16$ neurons total; fusiform, multipolar, and pyramidal: 
observed between lamina I neurons and other neuronal types could not be accounted for by our experimental paradigm, as in parallel recordings from hippocampal CA1 neurons we measured calcium responses consistent with those reported previously. Thus, spinal cord lamina I neurons have unique AP-evoked calcium kinetics, which are especially slow to rise and decay within the nucleus.

The kinetics of responses within the different compartments were consistent across the subpopulations of neurons when categorized either by firing type or by morphologic category. The similarity of the kinetics in each compartment, across the subpopulations, was unanticipated as the different firing types and morphologies roughly correspond to different functional types of lamina I neurons. In particular, as excitatory neurons are mainly delayed onset (Yasaka et al., 2010), and tonic firing neurons are predominantly inhibitory (Punnakkal et al., 2014), this allowed for an indirect comparison of these two populations. However, we found no significant differences in kinetics between these two firing types. Thus, we conclude that even neurons with opposing functions within lamina I have similar AP-evoked calcium responses within the soma and nucleus, and which invade the dendritic arbor.

\section{Dendritic and dendritic spine calcium responses are generated by actively backpropagating APs (bAPs)}

When we investigated the extent of calcium response propagation in the dendritic arbor, we found that calcium responses were present in all investigated dendrites and dendritic spines at all measured points within the dendritic arbor. Given the relatively slow rate at which calcium freely diffuses in cytosol, calcium responses produced solely by diffusion would have a lower response amplitude and slower time to peak the farther away from the soma they are measured (Sala and Hernandez-Cruz, 1990; Stuart and Hausser, 1994; Spruston et al., 1995). For example, uncaging calcium in dendrites and monitoring diffusion of calcium ions in the dendrites without activation of VGCCs yields a diffusion rate of $\sim 0.8 \mu \mathrm{m} / \mathrm{ms}$ (Korkotian and Segal, 2006). Therefore, calcium would require $100 \mathrm{~ms}$ to begin to reach dendritic segments $80 \mu \mathrm{m}$ away from the soma.

However, when we investigated AP-evoked calcium responses along the length of primary dendrites, we found that the beginning of the rise, the time to peak, and the peak response amplitude did not decrease with distance away from the soma. Our findings thus indicate backpropagation of the calcium responses through regenerative ionic conductances (i.e., bAPs), within the dendritic arbor. That the dendritic calcium responses were eliminated by blockers of VGCCs is consistent with bAPs mediated either as regenerative calcium spikes or as regenerative sodium spikes which drive the opening of VGCCs secondarily. This capacity of lamina I neurons to produce actively propagating bAPs gives them dendritic computational power, negating the

\section{$\leftarrow$}

$n=2, n=11$, and $n=3$ neurons, respectively. $I$, Mean peak response. Kruskal-Wallis oneway ANOVAs for somatic cytosol $p=0.906$, for nucleus $p=0.751$, for dendrite $p=0.092$, for dendritic spine $p=0.607$. J, Time to peak. Kruskal-Wallis one-way ANOVAs for somatic cytosol $p=0.715$, for nucleus $p=0.375$, for dendrite $p=0.168$, for dendritic spine $p=0.479$. $K$, Decay time constant $(\tau)$. Kruskal-Wallis one-way ANOVAs for somatic cytosol $p=0.551$, for nucleus $p=0.808$, for dendrite $p=0.699$, for dendritic spine $p=0.373$. $L$, AUC. KruskalWallis one-way ANOVAs for somatic cytosol $p=0.413$, for nucleus $p=0.860$, for dendrite $p=0.866$, for dendritic spine $p=0.602$. Unclassified neurons were not included for analysis. Data are mean \pm SEM. ${ }^{*} p<0.05 .{ }^{* * *} p<0.005$. common wisdom that dorsal horn dendrites simply serve to convey information to the soma.

When we compared dendritic spine calcium responses with dendritic calcium responses, we found no increase in time to peak and no delay in calcium response initiation within the dendritic spine compartment. Previous studies have found that dendritic spine neck resistance in cortical and hippocampal neurons is sufficiently high that there is negligent passive diffusion of calcium (Sabatini and Svoboda, 2000; Sabatini et al., 2001). Therefore, if dendritic spines in lamina I neurons have neck resistance similar to those in cortical and hippocampal neurons, the observed calcium responses in dendritic spines strongly indicate the presence of VGCCs in the dendritic spines of lamina I neurons.

Our findings indicate that somatic APs in lamina I neurons drive calcium responses across all dendritic spines in the neuron, not just those that provided depolarization to reach AP threshold. Thus, calcium-dependent processes could also occur in spines that had not been activated, giving a potential mechanism for heterosynaptic plasticity, as previously observed in the spinal dorsal horn (Woolf and Salter, 2000). The presence of bAPs in lamina I neurons is also relevant to a form of homosynaptic potentiation known as spike-timing-dependent plasticity, which has been observed in these neurons (Li and Baccei, 2016). Calcium responses elicited at dendritic spines activated by glutamatergic synaptic input may differ from those we observed here due to calcium entry via NMDARs or AMPARs lacking the GluA2 subunit. Future studies using glutamate uncaging may help to determine how such activation of glutamate receptors affects the resultant AP-evoked calcium response.

\section{Calcium responses in lamina I neurons encode AP firing in lamina I neurons}

We found that calcium responses are able to encode information about the number of APs fired in a burst and, to a lesser extent, the frequency of AP firing. In other neuronal types, calcium responses summate linearly to the number of APs fired, and supralinearly at higher-frequency firing, especially within their dendrites (Power and Sah, 2002; Liao and Lien, 2009; Tada et al., 2014). However, we did not find supralinear summation of responses at any frequency or number of APs in lamina I neurons. Instead, we found sublinear summation, which was dependent on the number of APs fired and, to a lesser extent, the frequency of firing. The AUC of the calcium response increased from 1-2 to 4 APs; however, no relationship was found when frequency increased, indicating that AUC is capable of tracking the number of APs fired, but not the frequency. Similarly, apart from the somatic cytosol, the maximum amplitude of the response largely increased with AP number, rather than with frequency.

Our findings also help to reconcile findings that calcium-dependent LTP of lamina I neurons can be obtained through electrical stimulation of presynaptic fibers over a wide range of frequencies ranging from 2 to $100 \mathrm{~Hz}$ (Sandkuhler and Liu, 1998; Terman et al., 2001; Yang et al., 2004; Fenselau et al., 2011; Ruscheweyh et al., 2011; Bonin and De Koninck, 2014; Luo et al., 2014). If calcium-dependent gene transcription is initiated by reaching a threshold intracellular calcium concentration, our findings suggest that the most important factor determining initiation of gene transcription is not the firing frequency, but rather the number of APs fired.

When we administered ryanodine onto a subset of neurons, we found evidence for CICR in response to AP firing. In other 
neuronal types, CICR contributes to calcium responses in the somatic cytosol, nuclei, and dendrites, boosting intracellular calcium concentration, and decreasing the threshold for gene transcription (Ronde and Nichols, 1997; Sandler and Barbara, 1999; van de Vrede et al., 2007; Goussakov et al., 2010; Bengtson and Bading, 2012). It has also been shown that blockade of ryanodine receptors in the spinal cord blocks induction of LTP, implying that CICR may be required for potentiation of lamina I neurons (Cheng et al., 2010; Drdla-Schutting et al., 2012; Lu et al., 2012; Ferrari et al., 2016). Thus, from our findings, we propose that calcium responses due to AP firing may be the critical link in various forms of synaptic plasticity within some populations of lamina I neuron.

In conclusion, we have identified the presence of AP-evoked calcium entry in lamina I neurons, providing the first evidence that single APs elicit calcium responses in the nucleus, somatic cytosol, dendrites, and dendritic spines of lamina I neurons. The amplitude of the calcium response is graded to the number of APs fired, providing a mechanism for AP firing to encode a relevant calcium signal. Calcium responses are dependent on VGCCs for initiation, and somatic calcium responses are boosted via CICR. Overall, these findings give insight into the excitability of lamina I neurons, and the means through which calcium is regulated during activity. These data also suggest that calcium accumulation in lamina I neurons is a potential mechanism for AP firing to initiate calcium-dependent gene transcription, which could lead to activity-dependent plasticity in these neurons.

\section{References}

Basbaum AI, Bautista D, Scherrer G, Julius D (2009) Cellular and molecular mechanisms of pain. Cell 139:267-284.

Bengtson CP, Bading H (2012) Nuclear calcium signaling. Adv Exp Med Biol 970:377-405.

Berridge MJ, Lipp P, Bootman MD (2000) The versatility and universality of calcium signalling. Nat Rev Mol Cell Biol 1:11-21.

Bloodgood BL, Sabatini BL (2007) Ca(2+) signaling in dendritic spines. Curr Opin Neurobiol 17:345-351.

Bonin RP, De Koninck Y (2014) A spinal analog of memory reconsolidation enables reversal of hyperalgesia. Nat Neurosci 17:1043-1045.

Bourinet E, Altier C, Hildebrand M, Trang T, Salter M, Zamponi G (2014) Calcium-permeable ion channels in pain signaling. Physiol Rev 94:81140.

Carter AG, Sabatini BL (2004) State-dependent calcium signaling in dendritic spines of striatal medium spiny neurons. Neuron 44:483-493.

Casale A, McCormick D (2011) Active Action Potential Propagation But Not Initiation in Thalamic Interneuron Dendrites. J. Neurosci 31:1828918302.

Cheng LZ, Lu N, Zhang YQ, Zhao ZQ (2010) Ryanodine receptors contribute to the induction of nociceptive input-evoked long-term potentiation in the rat spinal cord slice. Mol Pain 6:1.

Chery N, Yu X, Koninck Y (2000) Visualization of lamina I of the dorsal horn in live adult rat spinal cord slices. J Neurosci Methods 96:133-142.

Cichon J, Gan WB (2015) Branch-specific dendritic $\mathrm{Ca}(2+)$ spikes cause persistent synaptic plasticity. Nature 520:180-185.

Cordero-Erausquin M, Allard S, Dolique T, Bachand K, Ribeiro-da-Silva A, De Koninck Y (2009) Dorsal Horn Neurons Presynaptic to Lamina I Spinoparabrachial Neurons Revealed by Transynaptic Labeling. J. Comp. Neurol 517:601-615.

Costigan M, Scholz J, Woolf CJ (2009) Neuropathic pain: a maladaptive response of the nervous system to damage. Annu Rev Neurosci 32:1-32.

Coull J, Boudreau D, Bachand K, Prescott S, Nault F, Sik A, De Koninck P, Koninck Y (2003) Trans-synaptic shift in anion gradient in spinal lamina I neurons as a mechanism of neuropathic pain. Nature 424:938-942.

Denk W, Yuste R, Svoboda K, Tank D (1996) Imaging calcium dynamics in dendritic spines. Curr Opin Neurobiol 6:372-378.
Doerner JF, Febvay S, Clapham D (2012) Controlled delivery of bioactive molecules into live cells using the bacterial mechanosensitive channel MscL. Nat Commun 3:990.

Doolen S, Blake CB, Smith BN, Taylor BK (2012) Peripheral nerve injury increases glutamate-evoked calcium mobilization in adult spinal cord neurons. Mol Pain 8:56.

Drdla-Schutting R, Benrath J, Wunderbaldinger G, Sandkühler J (2012) Erasure of a spinal memory trace of pain by a brief, high-dose opioid administration. Science 335:235-238.

Evstratova A, Chamberland S, Topolnik L (2011) Cell type-specific and activity-dependent dynamics of action potential-evoked $\mathrm{Ca}^{2+}$ signals in dendrites of hippocampal inhibitory interneurons. J Physiol 589:1957-1977.

Fekete A, Johnston J, Delaney K (2014) Presynaptic T-type $\mathrm{Ca}^{2+}$ channels modulate dendrodendritic mitral-mitral and mitral-periglomerular connections in mouse olfactory bulb. J Neurosci 34:14032-14045.

Fenselau H, Heinke B, Sandkuhler J (2011) Heterosynaptic long-term potentiation at GABAergic synapses of spinal lamina I neurons. J Neurosci 31:17383-17391.

Ferrari LF, Khomula EV, Araldi D, Levine JD (2016) Marked sexual dimorphism in the role of the ryanodine receptor in a model of pain chronification in the rat. Sci Rep 6:31221.

Galhardo V, Lima D (1999) Structural Characterization of Marginal (Lamina I) Spinal Cord Neurons in the Cat: A Golgi Study. J. Comp. Neurol 414:315-333.

Gauriau C, Bernard JF (2002) Pain pathways and parabrachial circuits in the rat. Exp Physiol 87:251-258.

Golding NL, Kath WL, Spruston N (2001) Dichotomy of action-potential backpropagation in CA1 pyramidal neuron dendrites. J Neurophysiol 86:2998-3010.

Goussakov I, Miller MB, Stutzmann GE (2010) NMDA-mediated Ca(2+) influx drives aberrant ryanodine receptor activation in dendrites of young Alzheimer's disease mice. J Neurosci 30:12128-12137.

Hardingham GE, Chawla S, Johnson CM, Bading H (1997) Distinct functions of nuclear and cytoplasmic calcium in the control of gene expression. Nature 385:260-265.

Higley MJ, Sabatini BL (2012) Calcium signaling in dendritic spines. Cold Spring Harb Perspect Biol 4:a005686.

Hildebrand ME, Mezeyova J, Smith PL, Salter MW, Tringham E, Snutch TP (2011) Identification of sodium channel isoforms that mediate action potential firing in lamina I/II spinal cord neurons. Mol Pain 7:67.

Hildebrand M, Pitcher G, Harding E, Li H, Beggs S, Salter M (2014) Glun2b and Glun2d NMDARs dominate synaptic responses in the adult spinal cord. Sci Rep 4:4094.

Ikeda H, Heinke B, Ruscheweyh R, Sandkuhler J (2003) Synaptic plasticity in spinal lamina I projection neurons that mediate hyperalgesia. Science 299:1237-1241.

Ji RR, Kohno T, Moore KA, Woolf CJ (2003) Central sensitization and LTP: do pain and memory share similar mechanisms? Trends Neurosci 26:696-705.

Jia H, Rochefort NL, Chen X, Konnerth A (2011) In vivo two-photon imaging of sensory-evoked dendritic calcium signals in cortical neurons. Nat Protoc 6:28-35.

Keller AF, Beggs S, Salter MW, De Koninck Y (2007) Transformation of the output of spinal lamina I neurons after nerve injury and microglia stimulation underlying neuropathic pain. Mol Pain 3:27.

Korkotian E, Segal M (2006) Spatially confined diffusion of calcium in dendrites of hippocampal neurons revealed by flash photolysis of caged calcium. Cell Calcium 40:441-449.

Kuczewski N, Porcher C, Ferrand N, Fiorentino H, Pellegrino C, Kolarow R, Lessmann V, Medina I, Gaiarsa JL (2008) Backpropagating action potentials trigger dendritic release of BDNF during spontaneous network activity. J Neurosci 28:7013-7023.

Kyrozis A, Albuquerque C, Gu J, MacDermott AB (1996) Ca(2+)-dependent inactivation of NMDA receptors: fast kinetics and high $\mathrm{Ca}^{2+}$ sensitivity in rat dorsal horn neurons. J Physiol 495:449-463.

Li J, Baccei ML (2016) Neonatal tissue damage promotes spike timing-dependent synaptic long-term potentiation in adult spinal projection neurons. J Neurosci 36:5405-5416.

Liao CW, Lien CC (2009) Estimating intracellular $\mathrm{Ca}^{2+}$ concentrations and buffering in a dendritic inhibitory hippocampal interneuron. Neuroscience 164:1701-1711. 
Lima D, Coimbra A (1986) A Golgi study of the neuronal population of the marginal zone (lamina I) of the rat spinal cord. J Comp Neurol 244:18.

Lu N, Cheng LZ, Zhang YQ, Lu BC, Li YQ, Zhao ZQ (2012) Involvement of ryanodine receptors in tetanic sciatic stimulation-induced long-term potentiation of spinal dorsal horn and persistent pain in rats. J Neurosci Res 90:1096-1104.

Luo C, Kuner T, Kuner R (2014) Synaptic plasticity in pathological pain. Trends Neurosci 37:343-355.

Luo C, Seeburg PH, Sprengel R, Kuner R (2008) Activity-dependent potentiation of calcium signals in spinal sensory networks in inflammatory pain states. Pain 140:358-367.

Mantyh P, Rogers S, Honore P, Allen B, Ghilardi J, Li J, Daughters R, Lappi D, Wiley R, Simone D (1997) Inhibition of hyperalgesia by ablation of lamina I spinal neurons expressing the substance P receptor. Science 278:275-279.

Maravall M, Mainen ZF, Sabatini BL, Svoboda K (2000) Estimating intracellular calcium concentrations and buffering without wavelength ratioing. Biophys J 78:2655-2667.

Markram H, Helm PJ, Sakmann B (1995) Dendritic calcium transients evoked by single back-propagating action potentials in rat neocortical pyramidal neurons. J Physiol 485 (Pt 1):1-20.

Meissner G (2017) The structural basis of ryanodine receptor ion channel function. J Gen Physiol 149:1065-1089.

Naka A, Gruber-Schoffnegger D, Sandkühler J (2013) Non-Hebbian plasticity at C-fiber synapses in rat spinal cord lamina I neurons. Pain 154: 1333-1342.

Nimchinsky EA, Sabatini BL, Svoboda K (2002) Structure and function of dendritic spines. Annu Rev Physiol 64:313-353.

Polgar E, Durrieux C, Hughes DI, Todd AJ (2013) A quantitative study of inhibitory interneurons in laminae I-III of the mouse spinal dorsal horn. PLoS One 8:e78309.

Power JM, Sah P (2002) Nuclear calcium signaling evoked by cholinergic stimulation in hippocampal CA1 pyramidal neurons. J Neurosci 22:3454-3462

Prescott SA, De Koninck Y (2002) Four cell types with distinctive membrane properties and morphologies in lamina I of the spinal dorsal horn of the adult rat. J Physiol 539:817-836.

Punnakkal P, von Schoultz C, Haenraets K, Wildner H, Zeilhofer HU (2014) Morphological, biophysical and synaptic properties of glutamatergic neurons of the mouse spinal dorsal horn. J Physiol 592:759-776.

Ronde P, Nichols RA (1997) 5-HT3 receptors induce rises in cytosolic and nuclear calcium in NG108-15 cells via calcium-induced calcium release. Cell Calcium 22:357-365.

Rozsa B, Zelles T, Vizi ES, Lendvai B (2004) Distance-dependent scaling of calcium transients evoked by backpropagating spikes and synaptic activity in dendrites of hippocampal interneurons. J Neurosci 24:661-670.

Ruscheweyh R, Sandkuhler J (2002) Lamina-specific membrane and discharge properties of rat spinal dorsal horn neurones in vitro. J Physiol 541:231-244.

Ruscheweyh R, Wilder-Smith O, Drdla R, Liu XG, Sandkühler J (2011) Long-term potentiation in spinal nociceptive pathways as a novel target for pain therapy. Mol Pain 7:20

Sabatini B, Maravall M, Svoboda K (2001) $\mathrm{Ca}^{2+}$ signaling in dendritic spines. Curr Opin Neurobiol 11:349-356.
Sabatini B, Svoboda K (2000) Analysis of calcium channels in single spines using optical fluctuation analysis. Nature 408:589-593.

Sabatini BL, Oertner TG, Svoboda K (2002) The life cycle of $\mathrm{Ca}(2+)$ ions in dendritic spines. Neuron 33:439-452.

Sala F, Hernandez-Cruz A (1990) Calcium diffusion modeling in a spherical neuron: relevance of buffering properties. Biophys J 57:313-324.

Sandkuhler J, Liu X (1998) Induction of long-term potentiation at spinal synapses by noxious stimulation or nerve injury. Eur J Neurosci 10:24762480.

Sandler V, Barbara J (1999) Calcium-induced calcium release contributes to action potential-evoked calcium transients in hippocampal CA1 pyramidal neurons. J Neurosci 19:4325-4336.

Sjöstrom PJ, Nelson SB (2002) Spike timing, calcium signals and synaptic plasticity. Curr Opin Neurobiol 12:305-314.

Spruston N, Schiller Y, Stuart G, Sakmann B (1995) Activity-dependent action potential invasion and calcium influx into hippocampal CA1 dendrites. Science 268:297-300.

Stuart G, Hausser M (1994) Initiation and spread of sodium action potentials in cerebellar Purkinje cells. Neuron 13:703-712.

Tada M, Takeuchi A, Hashizume M, Kitamura K, Kano M (2014) A highly sensitive fluorescent indicator dye for calcium imaging of neural activity in vitro and in vivo. Eur J Neurosci 39:1720-1728.

Terman GW, Eastman CL, Chavkin C (2001) Mu opiates inhibit long-term potentiation induction in the spinal cord slice. J Neurophysiol 85:485494.

Tonini R, Ferraro T, Sampedro-Castaneda M, Cavaccini A, Stocker M, Richards CD, Pedarzani P (2013) Small-conductance $\mathrm{Ca}^{2+}$-activated $\mathrm{K}^{+}$ channels modulate action potential-induced $\mathrm{Ca}^{2+}$ transients in hippocampal neurons. J Neurophysiol 109:1514-1524.

Tsien R, Pozzan T (1989) Measurement of cytosolic free $\mathrm{Ca}^{2+}$ with quin2. Methods Enzymol 172:230-262.

van de Vrede Y, Fossier P, Baux G, Joels M, Chameau P (2007) Control of IsAHP in mouse hippocampus CA1 pyramidal neurons by RyR3-mediated calcium-induced calcium release. Pflugers Arch 455:297-308.

Waters J, Schaefer A, Sakmann B (2005) Backpropagating action potentials in neurones: measurement, mechanisms and potential functions. Progress in Biophysics and Molecular Biology 87:145-170.

Williams SR, Stuart GJ (2000) Action potential backpropagation and somato-dendritic distribution of ion channels in thalamocortical neurons. J Neurosci 20:1307-1317.

Woolf C, Salter M (2000) Neuronal plasticity: increasing the gain in pain. Science 288:1765-1769.

Yang HW, Hu XD, Zhang HM, Xin WJ, Li MT, Zhang T, Zhou LJ, Liu XG (2004) Roles of CaMKII, PKA, and PKC in the induction and maintenance of LTP of C-fiber-evoked field potentials in rat spinal dorsal horn. J Neurophysiol 91:1122-1133.

Yasaka T, Tiong SY, Hughes DI, Riddell JS, Todd AJ (2010) Populations of inhibitory and excitatory interneurons in lamina II of the adult rat spinal dorsal horn revealed by a combined electrophysiological and anatomical approach. Pain 151:475-488.

Yasuda R, Sabatini B, Svoboda K (2003) Plasticity of calcium channels in dendritic spines. Nat Neurosci 6:948-955. 